\title{
FOXM1 is a critical driver of lung fibroblast activation and fibrogenesis
}

\author{
Loka R. Penke, ${ }^{1}$ Jennifer M. Speth, ${ }^{1}$ Vijaya L. Dommeti, ${ }^{2}$ Eric S. White, ${ }^{1}$ Ingrid L. Bergin, ${ }^{3}$ and Marc Peters-Golden ${ }^{1}$ \\ 'Division of Pulmonary and Critical Care Medicine, Department of Internal Medicine, ${ }^{2}$ Michigan Center for Translational Pathology, Department of Pathology, and ${ }^{3}$ Unit for Laboratory Animal Medicine, \\ University of Michigan, Ann Arbor, Michigan, USA.
}

\begin{abstract}
While the transcription factor forkhead box M1 (FOXM1) is well known as a proto-oncogene, its potential role in lung fibroblast activation has never been explored. Here, we show that FOXM1 is more highly expressed in fibrotic than in normal lung fibroblasts in humans and mice. FOXM1 was required not only for cell proliferation in response to mitogens, but also for myofibroblast differentiation and apoptosis resistance elicited by TCF- $\beta$. The lipid mediator PGE $_{2}$, acting via cAMP signaling, was identified as an endogenous negative regulator of FOXM1. Finally, genetic deletion of FOXM1 in fibroblasts or administration of the FOXM1 inhibitor Siomycin A in a therapeutic protocol attenuated bleomycin-induced pulmonary fibrosis. Our results identify FOXM1 as a driver of lung fibroblast activation and underscore the therapeutic potential of targeting FOXM1 for pulmonary fibrosis.
\end{abstract}

\section{Introduction}

Substantial morbidity and mortality are attributable to fibroproliferative disorders that impair function of vital organs (1). Fibrotic remodeling of the lung parenchyma is observed in a variety of interstitial lung diseases, the most common of which is idiopathic pulmonary fibrosis (IPF). This is a chronic, irreversible, and progressive disease of unknown cause, which annually kills more people in the US than does breast cancer. Only recently has the FDA approved the first 2 drugs for the treatment of IPF, nintedanib and pirfenidone (2). Although these agents have been shown to slow progression of disease, neither of them reverses existing fibrosis. Thus, there remains a pressing unmet need to identify new molecular pathways driving fibrosis that can be targeted therapeutically. Because fibrosis is characterized by the accumulation of activated mesenchymal cells termed myofibroblasts, which elaborate extracellular matrix proteins, such as collagen (3), pathways responsible for the activation of these key effector cells are of particular interest.

Forkhead box M1 (FOXM1) is a transcription factor best recognized as a master regulator of cell cycle progression on the basis of studies in a variety of cell types, including epithelial cells, T cells, B cells, and dendritic cells $(4,5)$. Among its isoforms, FOXM1b (heretofore designated simply FOXM1) has been studied most extensively and is considered a proto-oncogene in a variety of human cancers $(6,7)$. FOXM1 regulates its own transcription as well as that of a number of cell cycle genes, including CCND1, involved in $\mathrm{G}_{1} / \mathrm{S}$ progression, and CCNB1 and PLK1, both of which are critical for $\mathrm{G}_{2} / \mathrm{M}$ transition. FOXM1 also upregulates BIRC5 (also known as survivin), a gene critical for mitotic spindle checkpoint integrity and for antiapoptotic activity (8-10).

Conflict of interest: The authors have declared that no conflict of interest exists

Submitted: March 22, 2016; Accepted: March 20, 2018

Reference information: J Clin Invest. 2018;128(6):2389-2405.

https://doi.org/10.1172/JCl87631.
However, the role of FOXM1 in driving activation of resident fibroblasts in the context of pulmonary fibrosis has never been explored. Additionally, little is known about the mechanisms by which FOXM1 is activated or the existence of negative endogenous regulators of its expression in any cell type. The data presented in this report provide new insights into the regulation of FOXM1 in fibrotic lung fibroblasts and support its potential as a target for therapeutic intervention in pulmonary fibrosis.

\section{Results}

Fibrotic fibroblasts exhibit increased FOXM1 expression. To evaluate the relevance of fibroblast FOXM1 in pulmonary fibrosis, we compared FOXM1 expression in fibrotic and control lung fibroblasts. Basal FOXM1 mRNA expression in lung fibroblasts isolated from patients with IPF was significantly (41\%) higher than that in controls, and greater expression was also manifested at the protein level (Figure 1, A and B). As has been observed previously for a number of parameters $(11,12)$, IPF fibroblasts exhibited a greater degree of heterogeneity than did control cells with respect to mRNA and protein expression of FOXM1. Of note, a good correlation was seen between mRNA and protein levels for individual IPF patients, and the 3 patients with low FOXM1 mRNA levels also exhibited low protein levels. Parallel studies were performed in fibroblasts grown from mouse lung harvested on day 21 following intrapulmonary administration of either saline or the fibrogenic substance bleomycin. Again, fibroblasts from bleomycin-treated lungs expressed significantly (32\%) higher Foxm1 mRNA and a corresponding higher level of FOXM1 protein than fibroblasts from control lungs (Figure $1, \mathrm{C}$ and D). Collectively, these data indicate that increased expression of FOXM1 is a feature of the highly activated lung fibroblasts that are the key effector cells in pulmonary fibrosis in both humans and a standard mouse model. We also evaluated the presence of FOXM1-positive cells in lung sections from control and fibrotic human and mouse lung via IHC. Normal human lung tissue and lung tissue from saline-treated mice showed positive staining in 
A

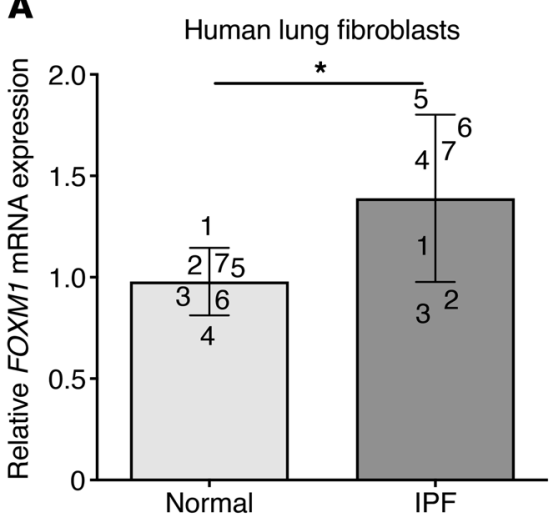

C

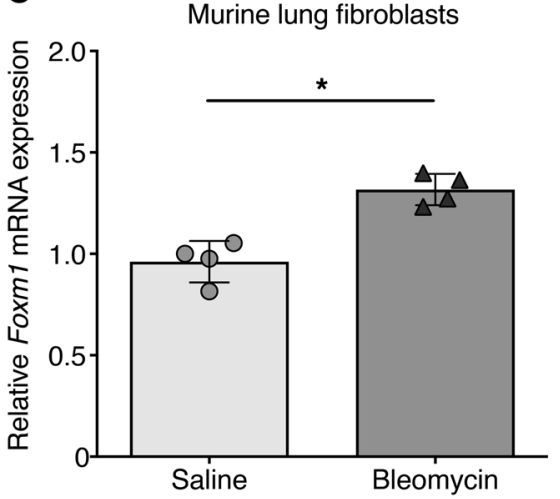

B

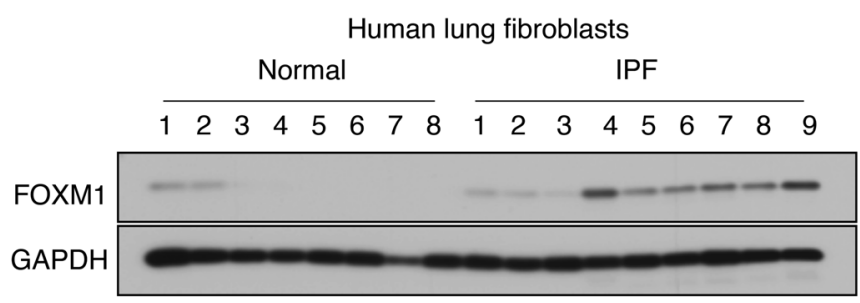

Figure 1. Fibrotic fibroblasts exhibit increased FOXM1 expression. (A and B) Basal FOXM1 expression in fibroblasts grown from lungs of patients with IPF and control nonfibrotic lungs by qPCR analysis (A) and Western blot analysis (B). (C and $\mathbf{D})$ Basal FOXM1 expression in fibroblasts grown from lungs of bleomycin- and saline-treated mice by qPCR analysis (C) and Western blot analysis (D). Each numeral in $\mathbf{A}$ and $\mathbf{B}$ denotes a single patient-derived cell line. In C, each symbol represents an individual murine-derived line of fibroblasts. Bars represent mean \pm SEM. In $\mathbf{B}$ and $\mathbf{D}$, each lane represents an individual patient- or murine-derived line of fibroblasts. ${ }^{*} P<0.05$. alveolar epithelium and alveolar macrophages, consistent with prior reports (13). Both IPF and bleomycin lung demonstrated abundant FOXM1 staining in the fibrotic interstitial regions. In addition, fibrotic foci within IPF lung demonstrated clear FOXM1 staining in elongated spindle-shaped cells that were also positive for Col1, consistent with fibroblasts/myofibroblasts (Supplemental Figure 1, A and B; supplemental material available online with this article; https://doi.org/10.1172/JCI87631DS1).

FOXM1 is critical for mitogen-induced fibroblast proliferation and expression of proliferation-associated genes. Although FOXM1 is known to regulate cell cycle events in various cell types, such a role in lung fibroblasts has never been reported. Since FGF2 and PDGF are pertinent fibroblast mitogens implicated in the pathogenesis of lung fibrosis (14), we assessed the role of FOXM1 induction in fibroblasts stimulated to proliferate in response to these growth factors. Based on its near-maximal stimulatory effect on the proliferation of commercially obtained normal human adult lung CCL210 fibroblasts at 48 hours (Supplemental Figure 2A), we chose an FGF2 concentration of $25 \mathrm{ng} / \mathrm{ml}$ for most of our subsequent studies. We confirmed that, at this dose, FGF2 induced the mRNA and protein expression of cell cycleregulated genes CCND1, CCNB1, PLK1, and BIRC5 at 24 to 48 hours (Supplemental Figure 2, B and C). We observed similar results with another mitogen, PDGF (Supplemental Figure 2, $\mathrm{D}$ and E). TGF- $\beta$ has also been reported to promote fibroblast proliferation (15), but we observed only a modest increase that reached statistical significance only at 5 to $10 \mathrm{ng} / \mathrm{ml}$ (Supplemental Figure $2 \mathrm{~F}$ ), with little effect at the dose sufficient to elicit myofibroblast differentiation $(2 \mathrm{ng} / \mathrm{ml})$. Stimulation of serum- starved fibroblasts with FGF2 elicited a time-dependent increase in FOXM1 expression (Figure 2A). This increase was completely blocked by pretreatment with the transcription inhibitor actinomycin D (Figure 2A). Figure 2B shows a corresponding increase in FOXM1 protein expression 48 hours after the addition of FGF2. In parallel with their respective effects on fibroblast proliferation, PDGF likewise increased FOXM1 expression, while TGF- $\beta$ ( 2 ng/ml) did not (Supplemental Figure $2 \mathrm{G})$.

Treatment with FGF2 elicited an increment in FOXM1 expression in nonfibrotic fibroblasts isolated from lungs resected from patients at our institution (Figure 2C) that was similar to its effect on commercially available CCL210 cells (Figure 2A). Although IPF fibroblasts exhibited higher levels of FOXM1 mRNA at baseline than did nonfibrotic controls (Figure 1A), they demonstrated a further increase in response to FGF2 stimulation; however, this increment was significantly (59.4\%) less than that seen in nonfibrotic controls (Figure 2C). As noted for basal FOXM1 mRNA expression in Figure 1A, the IPF cells exhibited a greater degree of variation in FGF2-stimulated levels of FOXM1 mRNA expression than did the controls. However, IPF lines with higher levels of FOXM1 demonstrated greater basal proliferation than did IPF lines with lower levels of FOXM1 (Figure 1A), whose proliferation rates were comparable to those of nonfibrotic controls (Figure 2D). A robust positive correlation between FOXM1 expression and proliferation rate for the entire group of patient-derived cell lines is shown in Supplemental Figure 3A.

Studies from various cancer cell lines of predominantly epithelial origin reported that growth factor-induced activation results in FOXM1 autoregulation through stimulation of its own 
A

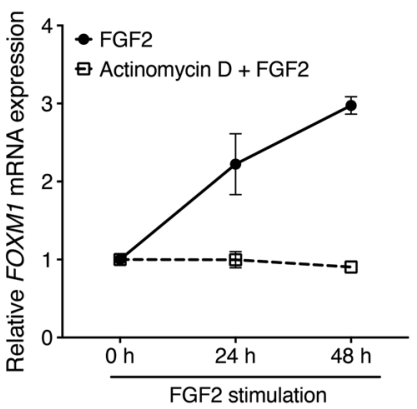

B

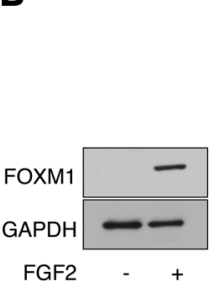

C

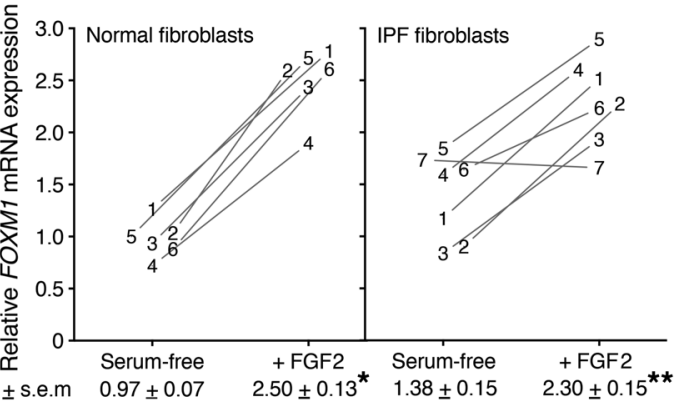

D

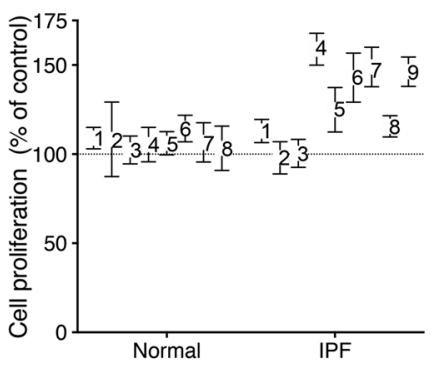

E

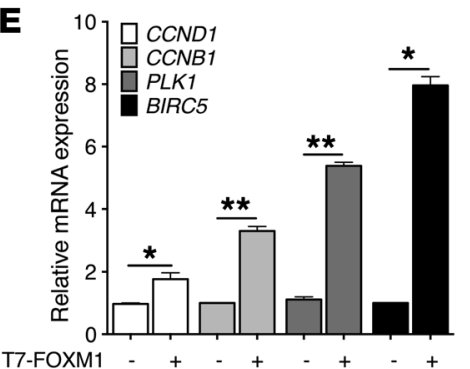

$\mathbf{F}$

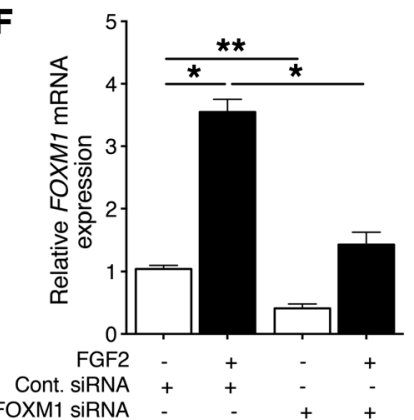

G
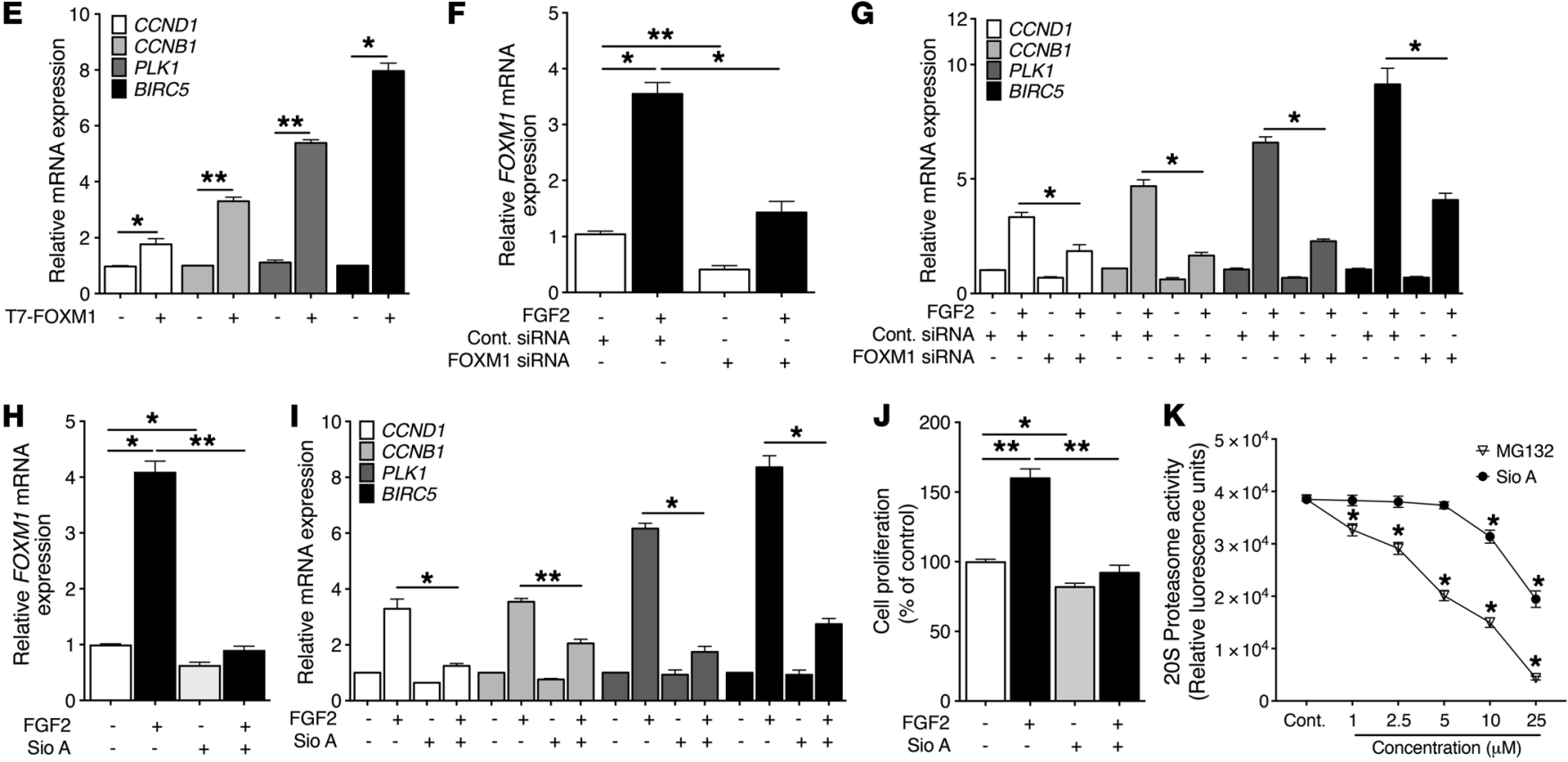

Figure 2. FOXM1 is critical for fibroblast proliferation. (A) Time-dependent induction of FOXM1 mRNA (analyzed by qPCR) in CCL210 cells by FGF2 stimulation in the presence and absence of actinomycin D. (B) Representative Western blot (from 1 of 3 independent experiments) of FOXM1 expression in CCL210 cells after 24 hours of treatment with and without FGF2. (C) qPCR analysis of FOXM1 mRNA expression in IPF and nonfibrotic fibroblasts treated for 48 hours with and without FGF2; cells from a given patient-derived line are denoted by a distinct numeral, and mean \pm SEM relative values are depicted below the graphs. ${ }^{*} P<0.05$; ${ }^{*} P<0.01$ vs. no FGF2 control, 2-tailed paired $t$ test. (D) Basal proliferation of fibroblasts from lungs of patients with IPF and control nonfibrotic lungs assayed by the CyQUANT NF Cell Proliferation Assay at 72 hours after culture. For $\mathbf{C}$ and $\mathbf{D}$, values are expressed relative to those of normal fibroblasts. (E) Expression of cell cycle-regulated genes CCND1, CCNB1, PLK1, and BIRC5 determined by qPCR in CCL210 cells transfected with FOXM1 overexpression plasmid or control plasmid. (F and $\mathbf{G}$ ) Effect of 24-hour pretreatment with FOXM1 or control (Cont) siRNA on FGF2-induced expression of FOXM1 (F) and cell cycle-regulated genes (G) as determined by qPCR. (H and I) Effect of treatment with $2.5 \mu \mathrm{M}$ Sio A on FGF2-induced expression of FOXM1 (H) and cell cycle-regulated genes (I). (J) Effect of Sio A on basal and FGF2-induced cell proliferation, as determined by the CyQUANT NF Cell Proliferation Assay at 72 hours in culture. (K) Effect of Sio A and the known proteasome inhibitor MG132 on proteasome activity in lung fibroblasts, as determined by the 205 proteasome activity assay. For $\mathbf{D}$ and $\mathbf{J}$, control value represents fluorescence value of cells initially seeded. ${ }^{*} P<0.05$; ${ }^{*} P<0.01,2$-way ANOVA.

transcription (8). To determine whether FOXM1 is sufficient to drive expression of cell cycle-regulated genes in CCL210 cells, we employed both CMV T7 promoter-driven and adenoviral-driven FOXM1 overexpression $(16,17)$ (Supplemental Figure 4, A-C). Indeed, forced overexpression of FOXM1 resulted in transcriptional activation of cell cycle-regulated genes (Figure 2E and Supplemental Figure 4D) and was potentiated by FGF2 stimulation (Supplemental Figure 4D). To test specifically whether FOXM1 is necessary for FGF2-induced expression of cell cycle-regulated genes, fibroblasts were transfected with FOXM1 siRNA prior to stimulation with FGF2. The degree of knockdown of basal FOXM1 with 24 hours of siRNA was approximately $56 \%$ (Figure 2 F). As compared with control scrambled siRNA, FOXM1 siRNA significantly attenuated the FGF2-induced increase in expression of cell cycle-regulated genes (Figure 2G).

We next tested the effects of Siomycin A (Sio A), an inhibitor of FOXM1 binding to DNA (18), on expression of FOXM1 and FOXM1 target genes as well as proliferation. Preliminary dosefinding experiments demonstrated substantial inhibitory effects of this agent at concentrations of 2.5 and $5 \mu \mathrm{M}$, and we employed the $2.5 \mu \mathrm{M}$ dose in subsequent experiments. Sio A significantly attenuated basal FOXM1 expression in fibroblasts (Figure $2 \mathrm{H}$ ) as well as the FGF2-induced upregulation of FOXM1 (Figure 2H) and of FOXM1 target genes (Figure 2I). As would be expected, Sio 
D

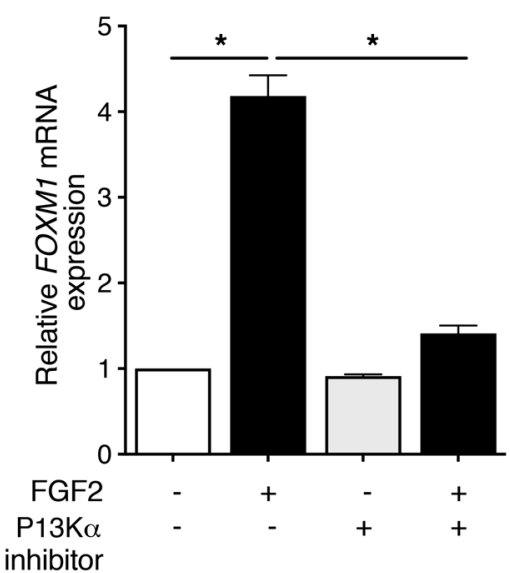

A

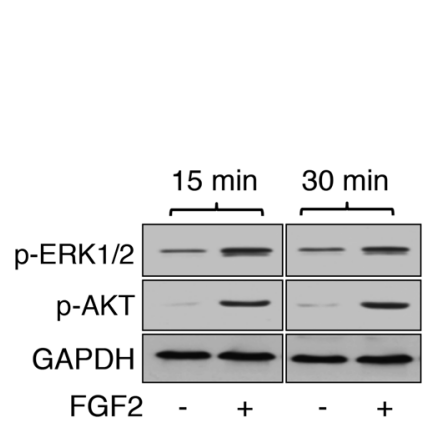

B

\section{FGF2
PI3K inhibitor \\ PDK1 inhibitor \\ AKT inhibitor \\ ERK $1 / 2$ inhibitor}

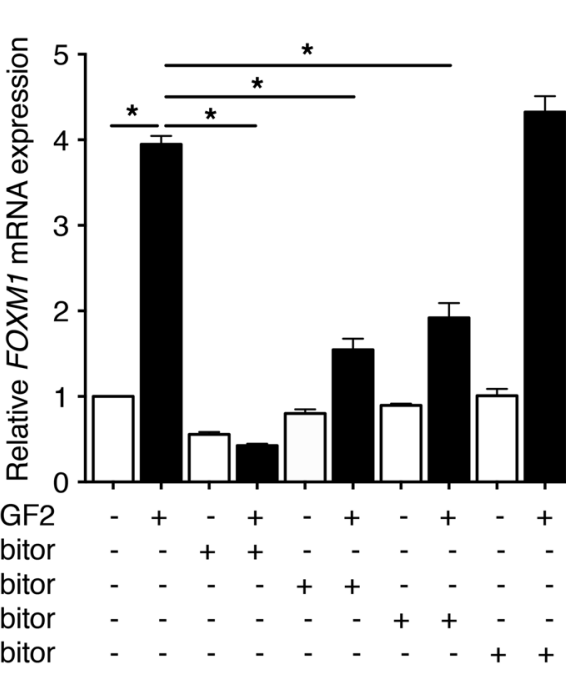

C

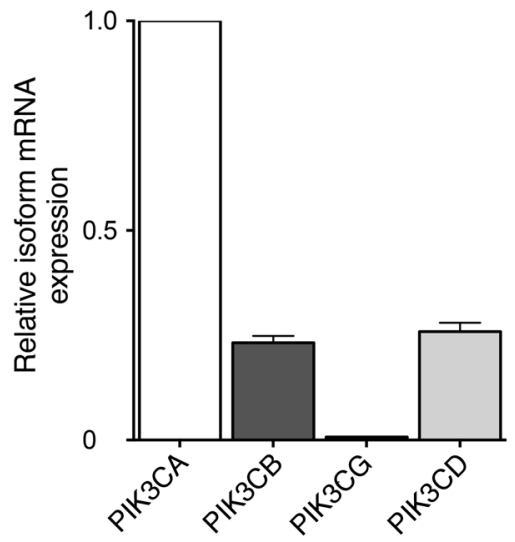

F
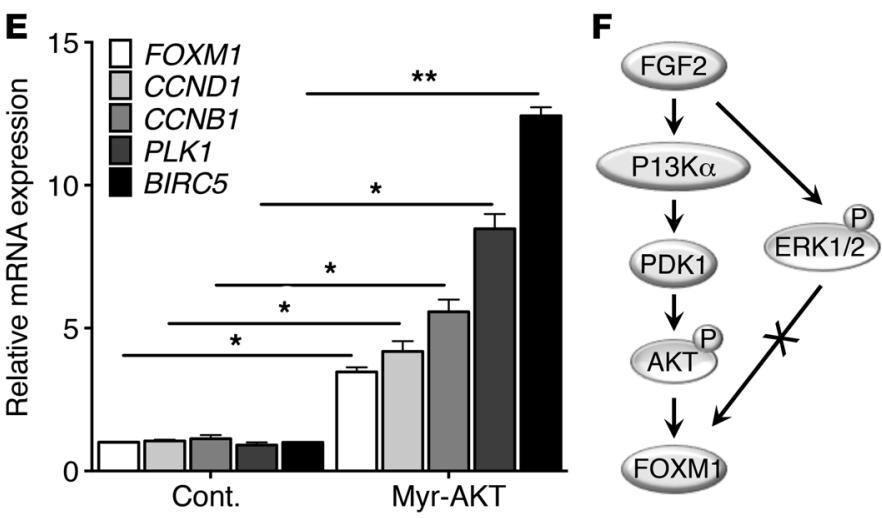

Figure 3. FGF2 upregulates FOXM1 expression via a PI3K/PDK1/AKT signaling pathway. (A) Representative Western blot (from 1 of 3 independent experiments) showing p-ERK1/2 and p-AKT in CCL210 cells stimulated with FGF2 for 15 minutes and 30 minutes. (B) qPCR analysis of FGF2-induced FOXM1 expression in cells pretreated with $10 \mu \mathrm{M} \mathrm{PI3K}$ inhibitor (LY294002), $1 \mu \mathrm{M}$ PDK1 inhibitor (GSK 2334470), $5 \mu \mathrm{M}$ AKT inhibitor (triciribine), or $5 \mu \mathrm{M}$ ERK1/2 inhibitor (U0126). (C) qPCR analysis of PIK3C isoform expression (relative to that of PIK3CA) in fibroblasts at baseline. (D) qPCR analysis of FGF2-induced FOXM1 expression in cells pretreated with and without $10 \mu \mathrm{M}$ PI3K $\alpha$ inhibitor. (E) mRNA expression of FOXM1 and cell cycle-regulated genes CCND1, CCNB1, PLK1, and BIRC5 in cells transfected with a Myr-AKT plasmid or control plasmid. ${ }^{*} P<0.05 ;{ }^{* *} P<0.01,2$-way ANOVA. (F) Schematic illustrating the signaling pathway by which FGF2 induces FOXM1 expression in fibroblasts.

A also markedly reduced proliferation in both unstimulated and FGF2-stimulated fibroblasts (Figure 2J). The $2.5 \mu \mathrm{M}$ concentration at which Sio A inhibited proliferation as well as expression of FOXM1 and cell cycle-regulated genes in lung fibroblasts was unassociated with demonstrable cytotoxicity (data not shown). Because Sio A has also been reported to inhibit activity of the proteasome (19), we directly examined its effect on proteasome activity in fibroblasts. As a positive control for inhibition in this assay, we employed the known proteasome inhibitor MG132 (20, 21). As expected, MG132 dose dependently inhibited proteasome activity, achieving near-complete inhibition at a concentration of $25 \mu \mathrm{M}$ (Figure 2K). Although Sio A elicited modest proteasome inhibition at doses of 10 and $25 \mu \mathrm{M}$, no such inhibitory activity was observed at 2.5 and $5 \mu \mathrm{M}$, the concentrations that were sufficient to inhibit FOXM1 expression and proliferation (22). In parallel experiments with BEAS-2B normal human bronchial epithelial cells, a Sio A dose of approximately $7.5 \mu \mathrm{M}$ was necessary to inhibit epidermal growth factor-induced proliferation (Supplemental Figure 6H).
Together, these data demonstrate that lung fibroblasts are highly sensitive to the cell cycle-inhibitory effects of Sio A and that such effects reflect inhibition of FOXM1 rather than of the proteasome.

FGF2 upregulates FOXM1 expression via a PI3Ka/PDK1/AKT pathway. To elucidate the molecular pathway involved in FGF2induced FOXM1 expression/activation, we focused on ERK1/2and AKT-signaling pathways, as both of these kinases were previously reported to activate FOXM1 (23). Indeed, both ERK1/2 and AKT exhibited phosphorylation upon FGF2 stimulation (Figure 3A). Inhibition of ERK1/2 did not prevent FGF2 actions on FOXM1 expression; however, inhibition of AKT or its upstream kinases PI3K and PDK1 markedly reduced FOXM1 expression (Figure 3B). Of the 4 isoforms of the PI3K catalytic subunit p110 (p110 $\alpha,-\beta,-\gamma$, and $-\delta$ ), fibroblasts predominantly expressed PIK3CA (encodes PI3K $\alpha$ ) (Figure 3C), and inhibition of PI3Ka likewise completely blocked FGF2-induced FOXM1 expression (Figure 3D). Forced overexpression of constitutively active AKT (Myr-AKT) (24) upregulated expression of FOXM1 and FOXM1 target genes in fibro- 
A

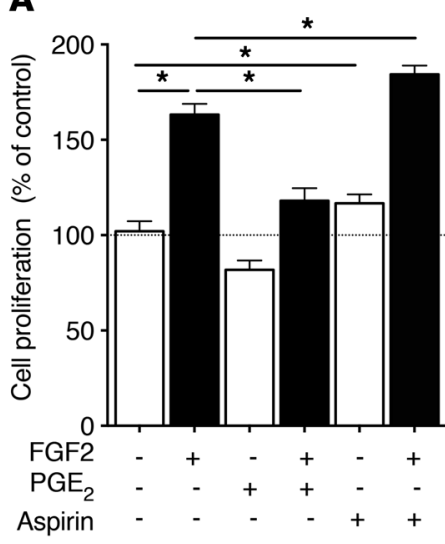

D

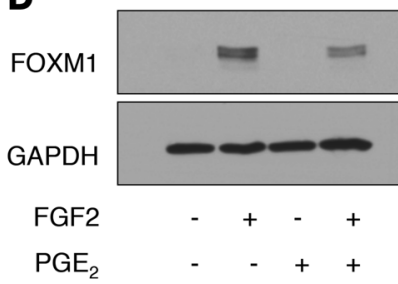

G

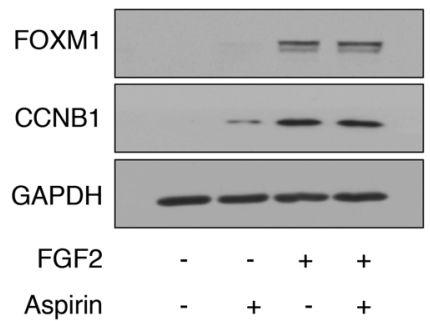

B

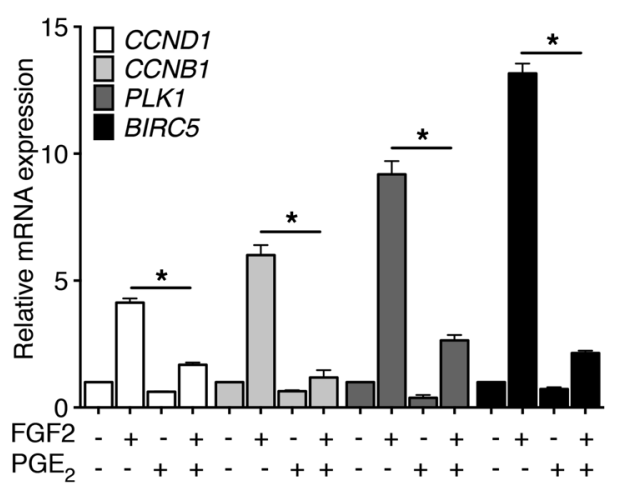

C

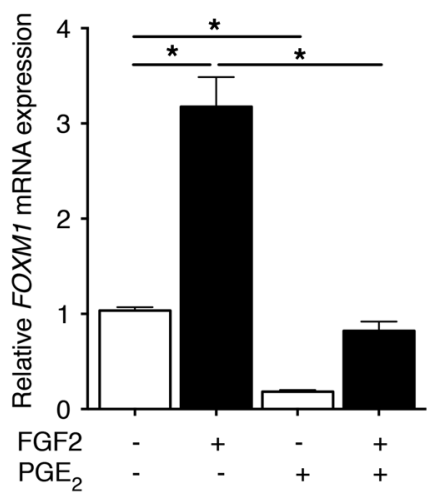

E

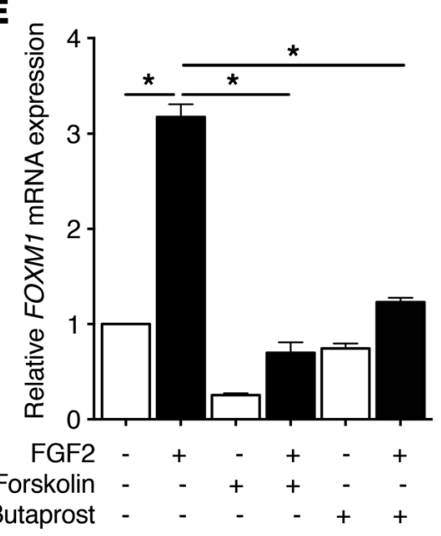

$\mathbf{F}$

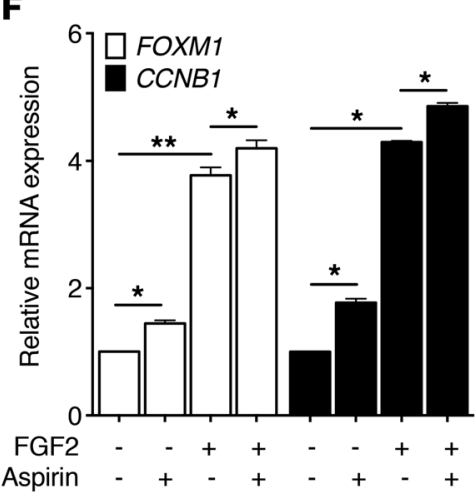

Figure 4. $\mathrm{PGE}_{2}$ acts as an endogenous brake for FOXM1 expression in lung fibroblasts. (A) Effect of pretreatment for 30 minutes with 0.1 mM aspirin or 0.5 $\mu \mathrm{M}$ PGE on FCF2-induced proliferation in CCL210 cells, as measured using the CyQUANT NF Cell Proliferation Assay at 72 hours. The control value represents the fluorescence value of cells initially seeded. (B and C) qPCR analysis of FGF2-induced expression of cell cycle-regulated genes (B) and FOXM1 (C) in cells pretreated for 30 minutes with and without $0.5 \mu \mathrm{M} \mathrm{PGE}_{2}$. (D) Representative Western blot showing basal and FGF2-induced FOXM1 expression in cells pretreated with and without $0.5 \mu \mathrm{M} P \mathrm{PE}_{2}$. (E) qPCR analysis of FGF2-induced FOXM1 expression in cells pretreated for 30 minutes with and without $10 \mu \mathrm{M}$ forskolin or $10 \mu \mathrm{M}$ butaprost. (F and $\mathbf{G )}$ Effect of pretreatment for 1 hour with $0.1 \mathrm{mM}$ aspirin on FGF2-induced expression of FOXM1 and CCNB1 evaluated by qPCR (F) and by Western blot (G). Western blots in $\mathbf{D}$ and $\mathbf{G}$ are representative of 3 independent experiments. ${ }^{*} P<0.05 ;{ }^{*} P<0.01,2$-way ANOVA.

blasts (Figure 3E). From these results, we conclude that PI3Ka signaling via PDK1/AKT, but not ERK1/2, mediates FGF2-induced FOXM1 upregulation in lung fibroblasts (Figure 3F).

$P G E_{2}$ acts as an endogenous brake for FOXM1 expression in lung fibroblasts. Previous studies have identified the endogenous lipid mediator $\mathrm{PGE}_{2}$ - the predominant prostanoid product of lung fibroblasts (25) - as a negative regulator of lung fibroblast proliferation, acting via its $\mathrm{G}$ protein-coupled receptor EP2 and cAMP signaling (26). However, the molecular machinery targeted by $\mathrm{PGE}_{2}$-cAMP to exert this inhibitory effect on proliferation remains unclear, and we therefore investigated the possibility that FOXM1 was a target for $\mathrm{PGE}_{2}$ regulation. Consistent with previous studies (26), exogenous $\mathrm{PGE}_{2}$ inhibited both basal and FGF2-induced fibroblast proliferation (Figure 4A). In contrast, irreversible inhibition of $\mathrm{PGE}_{2}$ synthesis with aspirin enhanced basal as well as FGF2-induced fibroblast proliferation (Figure 4A), supporting the conclusion that $\mathrm{PGE}_{2}$ represents an endogenous brake for lung fibroblast proliferation. Consistent with this role, $\mathrm{PGE}_{2}$ also inhibit- ed the upregulation of cell cycle-regulated genes induced by FGF2 (Figure 4B). We next examined the effects of PGE 2 on FOXM1 expression and found that $\mathrm{PGE}_{2}$ significantly reduced basal as well as FGF2-induced FOXM1 mRNA and protein expression (Figure 4, C and D). Similar actions were observed for butaprost (a PGE analog with selective EP2 receptor agonist activity) and the direct adenylyl cyclase activator forskolin (Figure 4E). Consistent with our observations with aspirin on fibroblast proliferation, aspirin treatment enhanced both basal and FGF2-induced FOXM1 expression as well as that of the cell cycle-regulated gene CCNB1 (Figure 4, F and G). From these findings, we conclude that endogenous and exogenous $\mathrm{PGE}_{2}$ via an EP2/cAMP pathway negatively regulates FOXM1 expression in parallel with its inhibition of lung fibroblast proliferation. We believe that these studies identify $\mathrm{PGE}_{2}$ as the first endogenous substance recognized as inhibiting FOXM1 activation/expression.

Molecular mechanisms for the inhibitory actions of $P G E_{2}$ on FOXM1. $\mathrm{PGE}_{2}$ has been reported to inhibit FGF2-mediated 
A

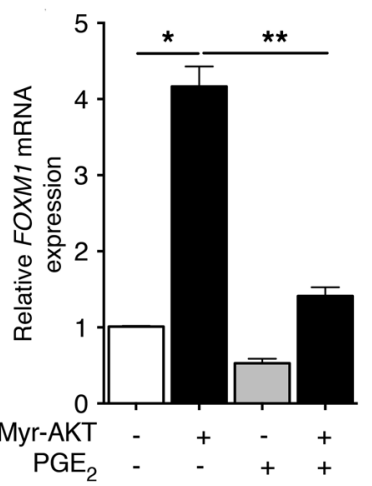

B

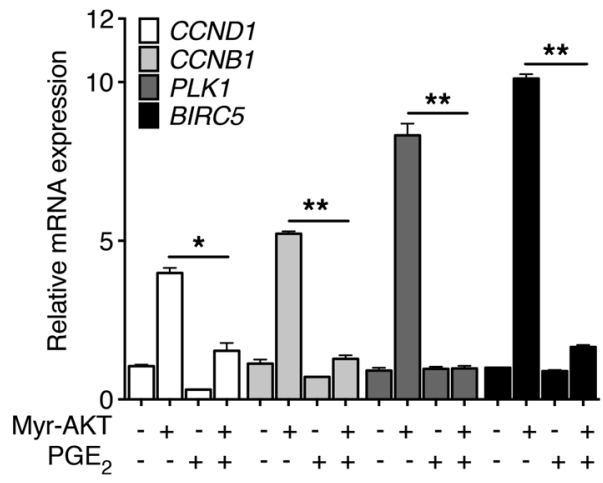

C

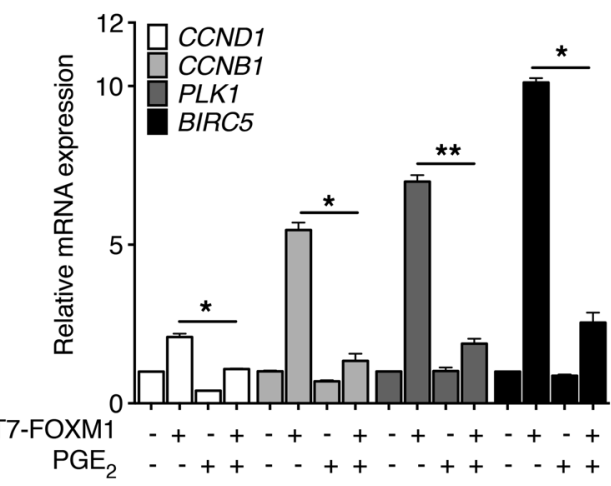

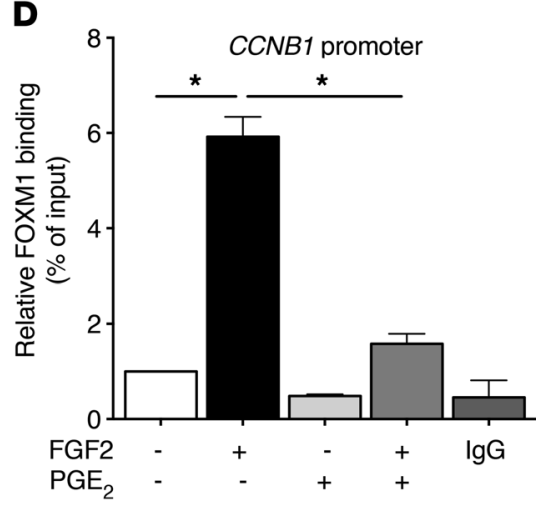
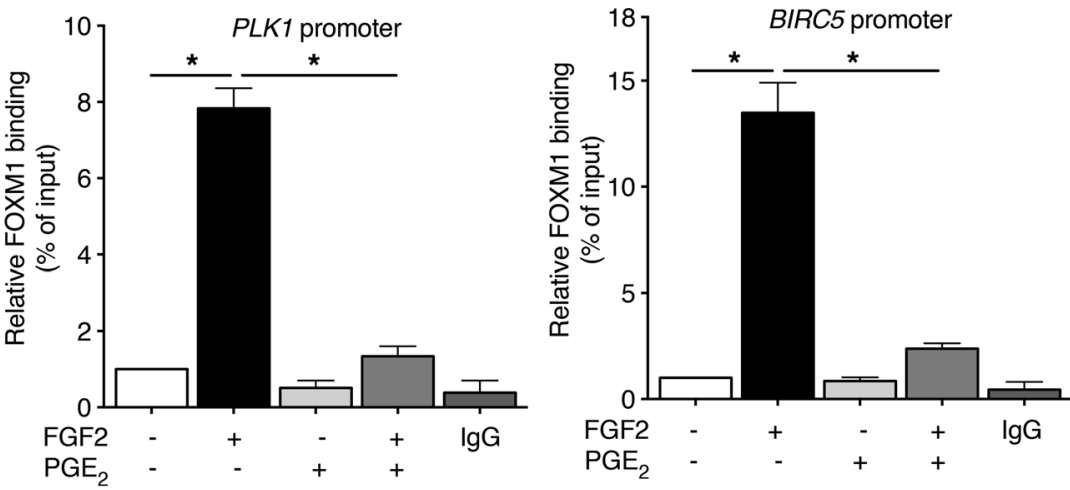

$\mathbf{E}$

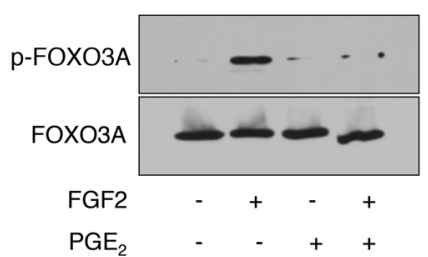

$\mathbf{F}$

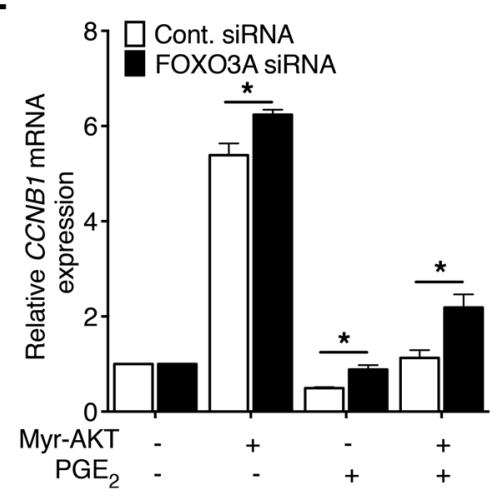

G

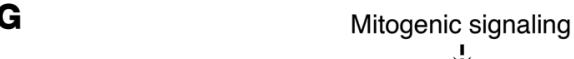

Mitogenic signaling

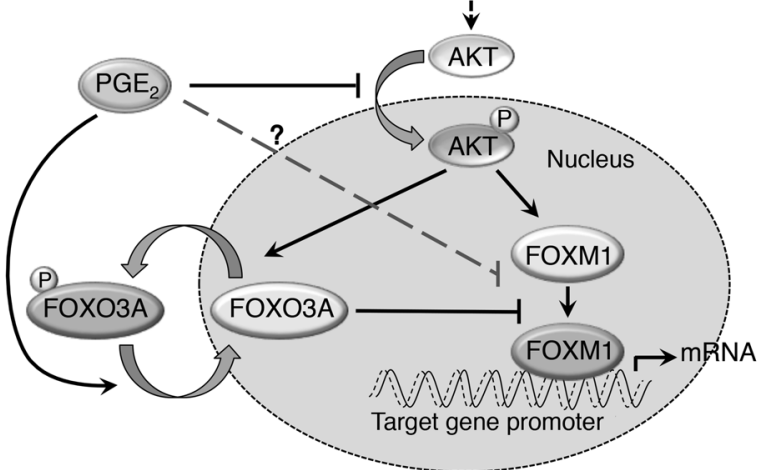

Figure 5. PGE 2 prevents FOXM1 binding to promoters of target genes. (A and B) CCL210 cells transfected with Myr-AKT plasmid for 24 hours and then treated with and without $0.5 \mu \mathrm{M}$ PGE for an additional 24 hours (A) or 48 hours (B) and analyzed by qPCR for FOXM1 expression (A) and expression of cell cycle-regulated genes CCND1, CCNB1, PLK1, and BIRC5 (B). (C) qPCR analysis of expression of cell cycle-regulated genes in cells transfected with FOXM1 overexpression plasmid for 24 hours and then treated with and without $0.5 \mu \mathrm{M} \mathrm{PGE}$ for an additional 48 hours. (D) Effects of 30-minute pretreatment with and without $0.5 \mathrm{M} \mathrm{PGE}_{2}$ on FGF2-induced FOXM1 binding to the CCNB1 (left), PLK1 (middle), and BIRC5 (right) promoters, determined by ChIP-qPCR

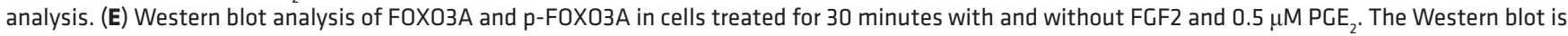
representative of 3 independent experiments. (F) Effect of prior transfection for 24 hours with FOXO3A or control siRNA on the ability of $0.5 \mu M P^{2} E_{2}$ to inhibit CCNB1 mRNA levels in cells expressing Myr-AKT. ${ }^{*} P<0.05$; ${ }^{*} P<0.01,2$-way ANOVA. (G) Schematic illustrating the multiple mechanisms by which $\mathrm{PCE}_{2}$ inhibits FOXM1 signaling in fibroblasts.

activation of AKT, and this action was implicated in its ability to inhibit FGF2-mediated migration of human fetal lung fibroblasts (27). We confirmed that $\mathrm{PGE}_{2}$ exerted a similar inhibitory effect on FGF2-induced AKT phosphorylation in adult CCL210 cells (Supplemental Figure 5A). In view of the critical role of AKT in the induction of FOXM1 in these cells (Figure 3), we anticipated that bypassing the PI3K-PDK1-mediated activation of AKT would overcome such upstream inhibitory effects of $\mathrm{PGE}_{2}$ on FOXM1 expression. Unexpectedly, forced expression of Myr-AKT failed to rescue the $\mathrm{PGE}_{2}$ inhibitory actions on expression of FOXM1 (Figure 5A) and FOXM1 target genes (Figure 5B). This suggests the possibility of additional $\mathrm{PGE}_{2}$ inhibitory mechanisms downstream of AKT phosphorylation/ activation. The inhibitory effects of $\mathrm{PGE}_{2}$ on expression of FOXM1 target genes were even preserved in the face of forced overexpression of FOXM1 (Figure 5C). We therefore considered 
A

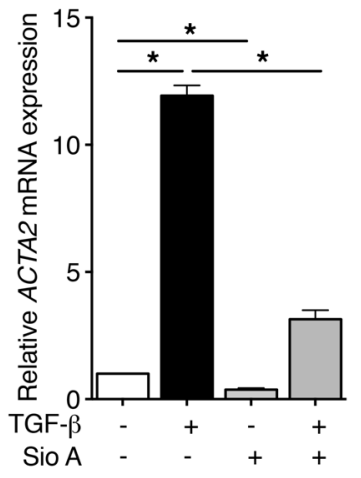

B

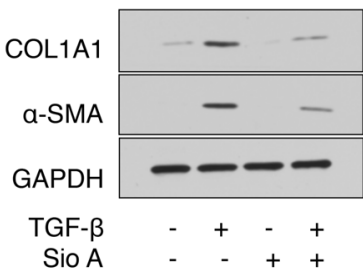

C

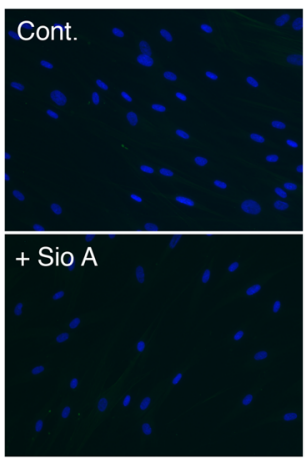

$\mathbf{F}$

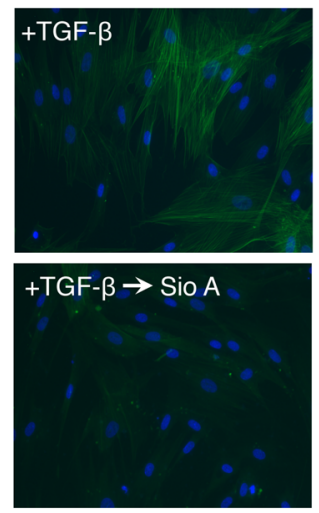

D

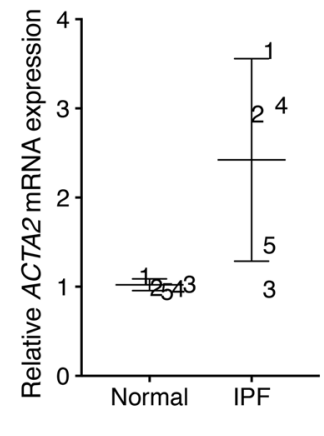

E

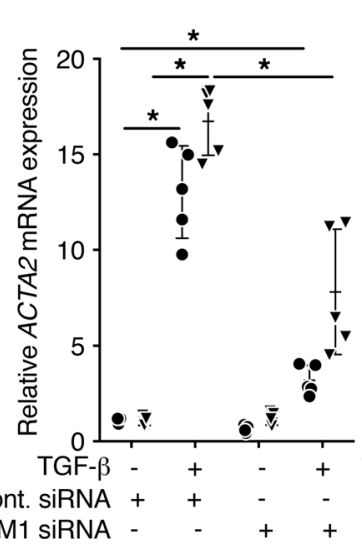

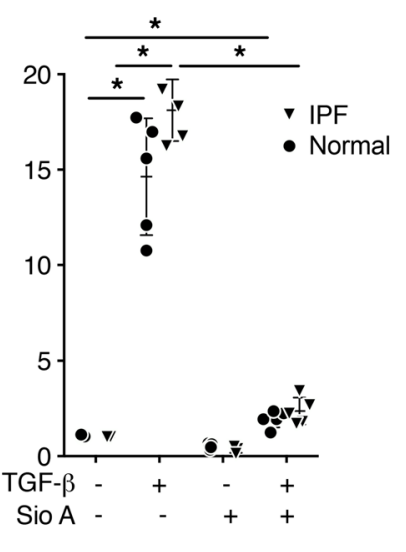

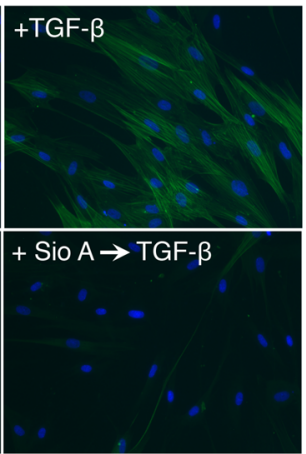

G

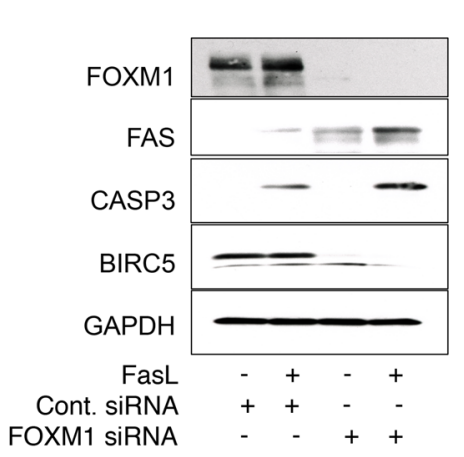

H

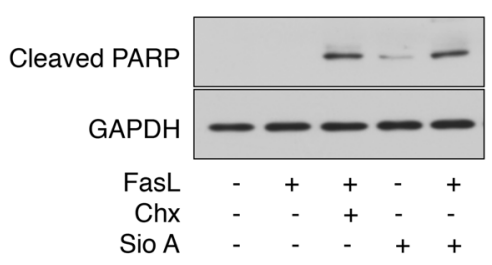

I

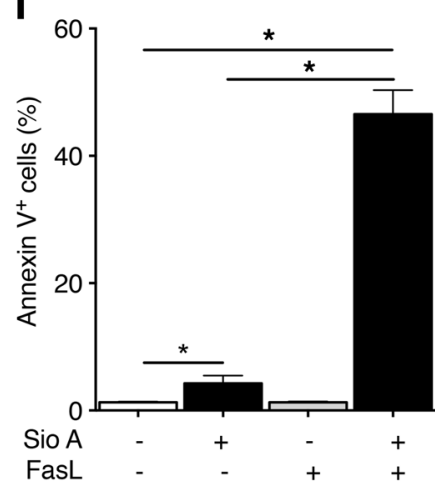

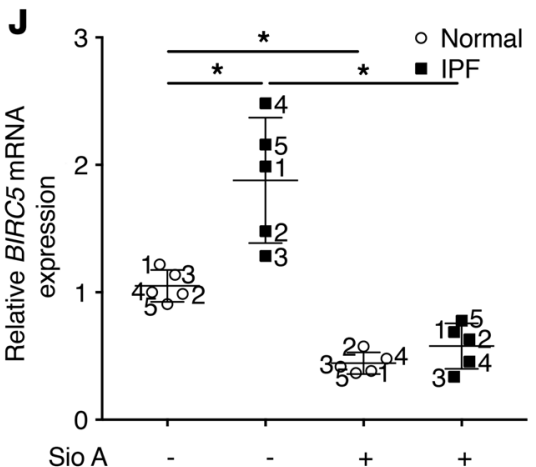

Figure 6. FOXM1 inhibition prevents and reverses TGF- $\beta$-induced myofibroblast differentiation and sensitizes myofibroblasts to FasL-induced apoptosis. (A-C) Effects of a 1-hour pretreatment of CCL210 cells with $2.5 \mu \mathrm{M}$ Sio A (prevention protocol) on TCF- $\beta$-induced expression of $\alpha$-SMA mRNA (A) and protein (B, Western blot; C, immunofluorescence microscopy) as well as collagen I protein (B). (D) Basal levels of ACTA2 mRNA in lung fibroblasts isolated from IPF patients or nonfibrotic controls $(n=5)$. (E) Effect of transfection with FOXM1 siRNA or scrambled (control) siRNA (for 16 hours) (left) or 30 -minute pretreatment with $2.5 \mu \mathrm{M}$ Sio A (right) on ACTA2 mRNA levels in IPF or control lung fibroblasts $(n=5)$ stimulated with and without TCF- $\beta$ for 24 hours.

(F) Immunofluorescence microscopic analysis of $\alpha$-SMA expression in TGF- $\beta$-generated myofibroblasts treated with $2.5 \mu \mathrm{M}$ Sio A for 24 hours (reversal protocol). (C) Effect of transfection with FOXM1 siRNA or scrambled (control) siRNA (for 16 hours) on FasL-induced apoptosis and expression of apoptosisassociated genes FAS, CASP3, and BIRC5 by Western blot analysis of TCF- $\beta$-generated myofibroblasts. (H and I) Effects of a 1-hour pretreatment with 2.5 $\mu \mathrm{M}$ Sio $\mathrm{A}$ on FasL-induced apoptosis in TCF- $\beta$-generated myofibroblasts, as determined by cleaved PARP expression assessed by Western blot (H) and by the frequency of annexin $\mathrm{V}$ staining assessed by flow cytometric analysis (I). (J) Effect of Sio A treatment for 24 hours on BIRC5 expression in nonfibrotic control and IPF fibroblasts. ${ }^{*} P<0.05$, 2-way ANOVA. Images in $\mathbf{C}$ and $\mathbf{D}$ are representative immunofluorescence images showing $\alpha$-SMA (green) and DAPI (blue). Original magnification, $\times 200$. Western blots in $\mathbf{B}, \mathbf{G}$, and $\mathbf{H}$ are representative of 3 independent experiments. ${ }^{*} P<0.05,2$-way ANOVA.

the possibility that $\mathrm{PGE}_{2}$ interfered with the binding of FOXM1 to promoter regions of DNA in its target genes. Consistent with our mRNA data (Figure 4B), ChIP-quantitative PCR (ChIPqPCR) confirmed that FGF2 stimulation increased the binding of FOXM1 to promoters in its target genes CCNB1, PLK1, and
BIRC5; such binding was, however, markedly disrupted by pretreatment with $\mathrm{PGE}_{2}$ (Figure 5D).

FOXO3A is another FOX family member known to antagonize FOXM1 activity. A recent study showed that IPF fibroblasts display low FOXO3A activity (28), and this is consistent with our 
A

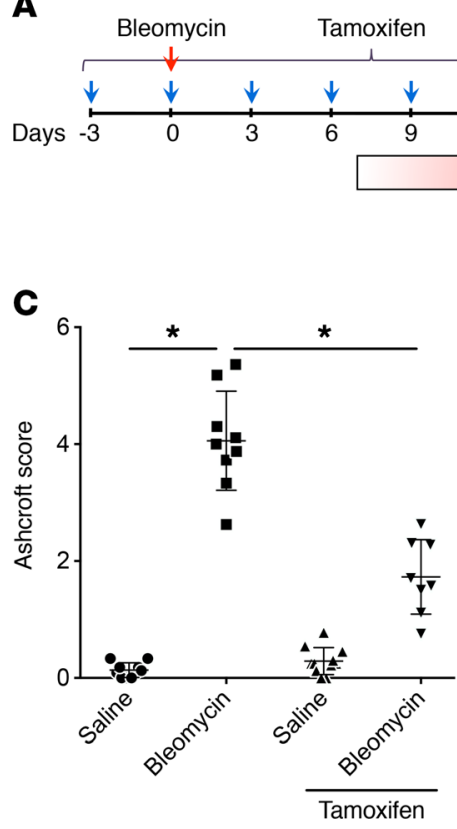

E

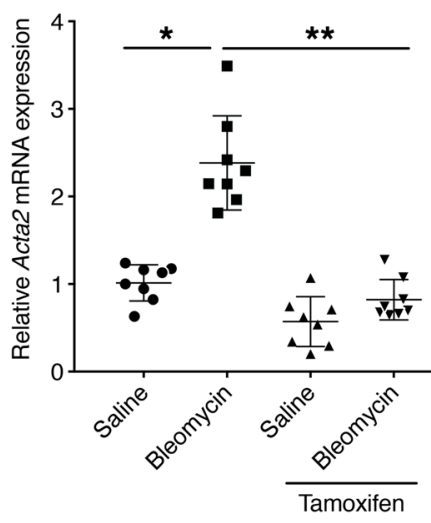

B

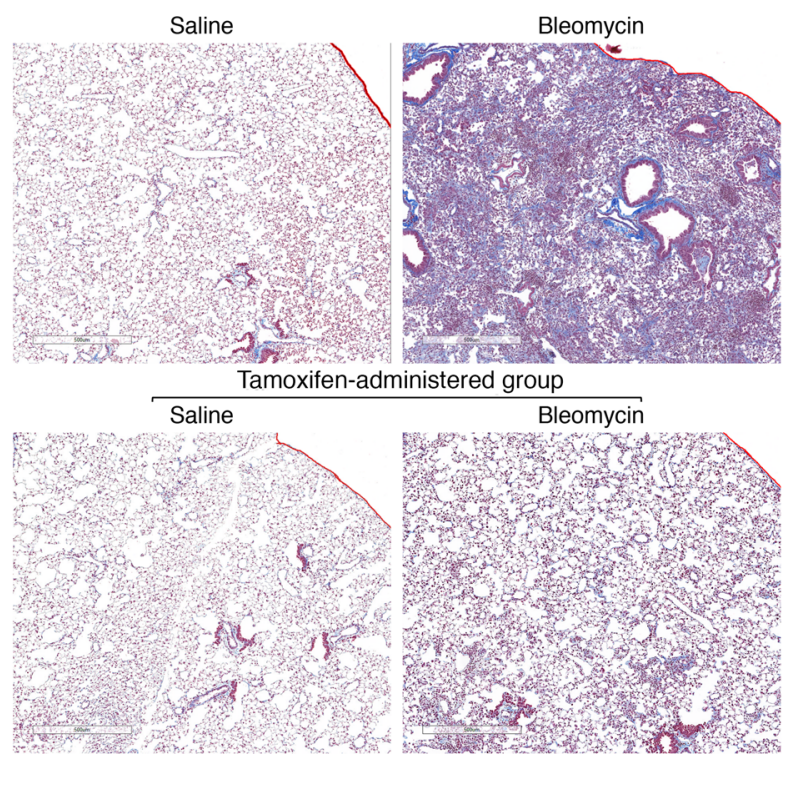

$\mathbf{F}$

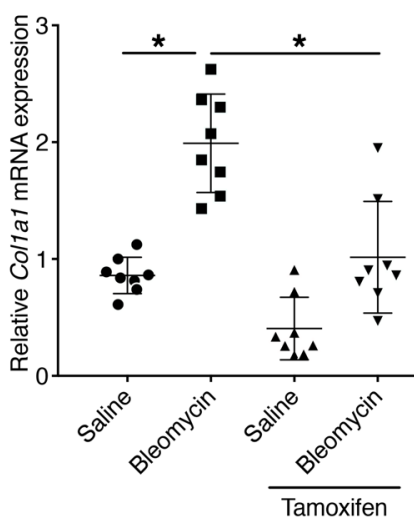

G

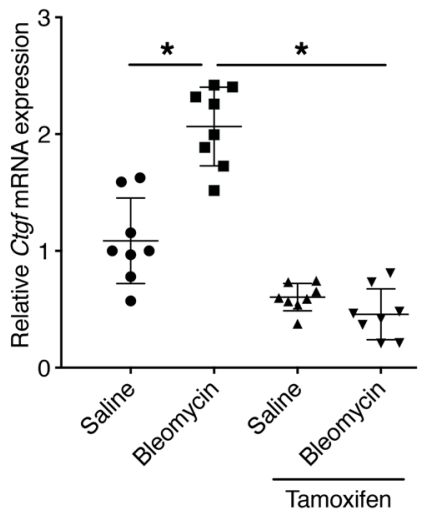

H

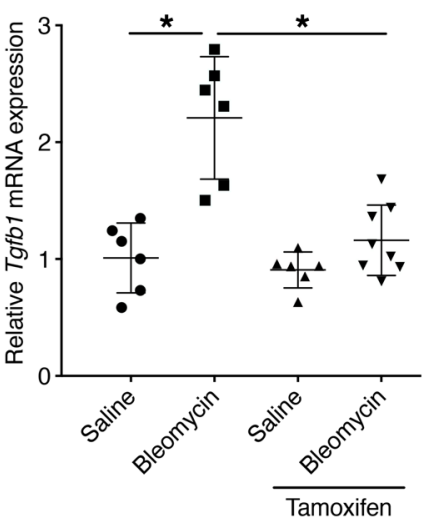

Figure 7. Fibroblast-specific deletion of FOXM1 in a prevention protocol protects mice from bleomycin-induced fibrosis. (A) Schematic illustrating the timelines for in vivo administration of tamoxifen and bleomycin and determination of experimental end points in the bleomycin model of pulmonary fibrosis. (B) Digital images of Masson's trichrome staining for collagen deposition (blue) at day 21. Original magnification, $\times 200$. Scale bars: $500 \mu \mathrm{m}$. (C-H) Effect of conditional fibroblast-specific FOXM1 deletion in mice treated with and without bleomycin, as reflected by changes in the Ashcroft histology score (C), lung hydroxyproline content (D), and the mRNA expression of fibrotic markers (Acta2, Col1a1, Ctgf, and Tgfb1) (E-H). In C-H, each symbol represents an individual mouse. Values in each group represent results from 2 pooled independent experiments with a total of 5 to 8 mice per group. ${ }^{*} P<0.05$; ${ }^{*} P<0.01$, 2-way ANOVA with Tukey's multiple comparisons test.

findings of high basal FOXM1 expression in fibrotic cells (Figure $1, \mathrm{~A}$ and $\mathrm{B})$. The antagonistic ability of $\mathrm{FOXO} 3 \mathrm{~A}$ requires that it be dephosphorylated and nuclear localized (29). During growthfactor stimulation, AKT-induced phosphorylation of FOXO3A drives it out of the nucleus (30), thus permitting active FOXM1 to bind to DNA. We confirmed that, as expected, stimulation with FGF2 promoted FOXO3A phosphorylation (Figure 5E). Consistent with its ability to diminish phosphorylated AKT (p-AKT) (Supplemental Figure 5A), PGE $_{2}$ also inhibited FOXO3A phosphorylation (Figure $5 \mathrm{E}$ ). In parallel with the phosphorylation status of FOXO3A, we also confirmed that FGF2 promoted, while $\mathrm{PGE}_{2}$ inhibited, its nuclear export (Supplemental Figure 5B). However, such activation of FOXO3A would not be expected to account for the inhibitory actions of $\mathrm{PGE}_{2}$ that we observed to exist downstream of AKT. Indeed, despite significant knockdown of FOXO3A mRNA and protein (Supplemental Figure 5, C and D), only a modest partial abrogation of the inhibitory effect of $\mathrm{PGE}_{2}$ on Myr-AKT-induced CCNB1 expression was observed (Figure $5 \mathrm{~F}$ ). This suggests some meaningful inhibitory action of $\mathrm{PGE}_{2}$ on FOXM1 DNA binding that is independent of FOXO3A. A schematic describing the diverse $\mathrm{PGE}_{2}$ inhibitory mechanisms on FOXM1 expression is presented in Figure $5 \mathrm{G}$.

Additional antifibrotic actions of Sio A and FOXM1 siRNA in TGF- $\beta$-induced myofibroblasts. The mesenchymal cells capable of elaborating large quantities of extracellular matrix proteins, such as collagens, that are most implicated in tissue fibrosis are myofi- 
A

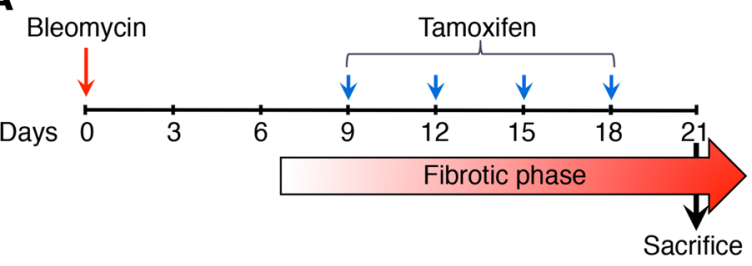

B

B Tamoxifen-administrated group

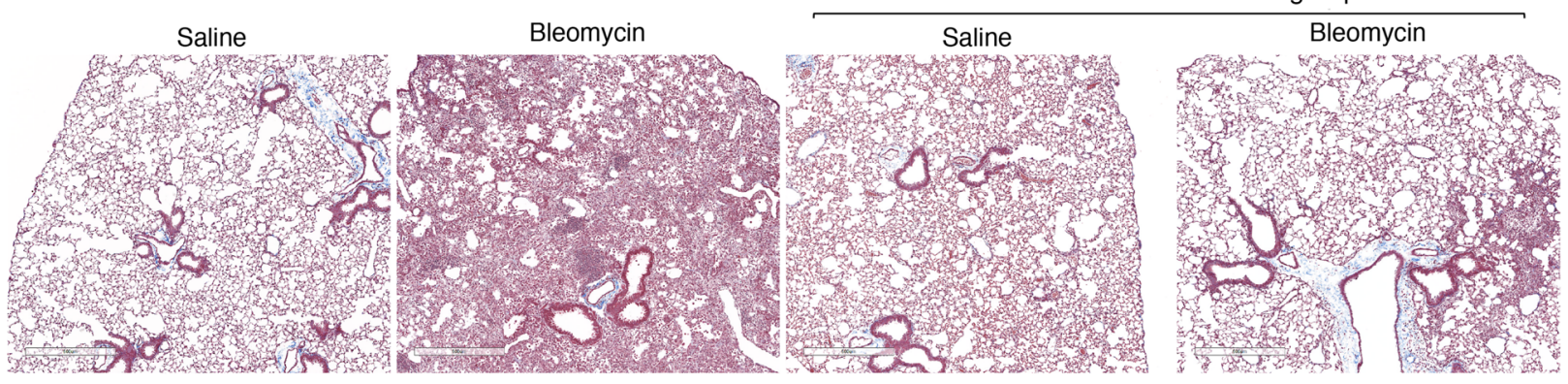

C

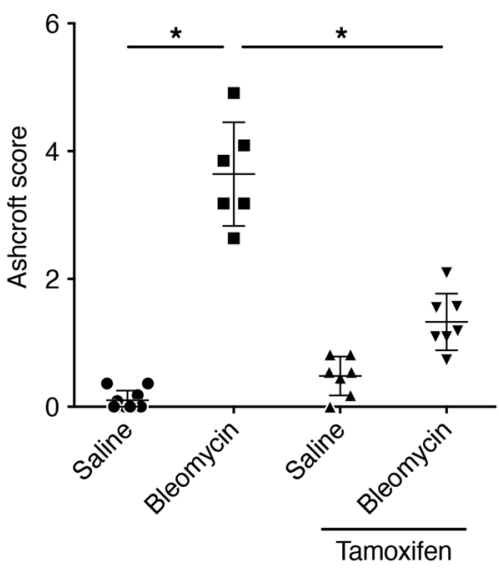

$\mathbf{E}$

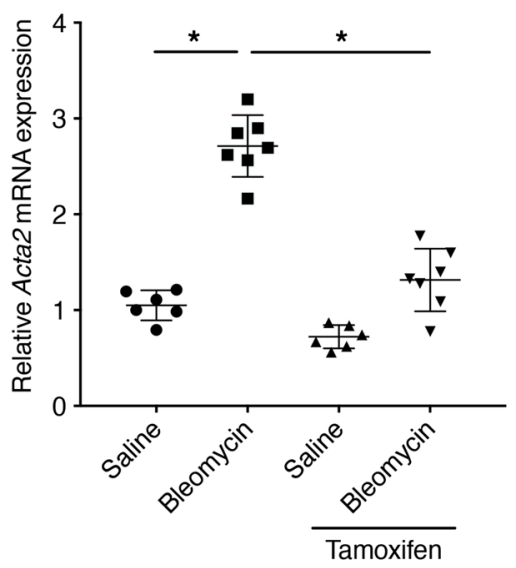

D

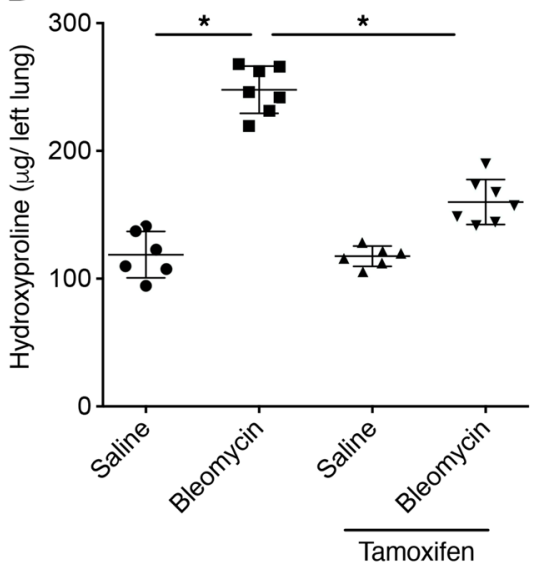

$\mathbf{F}$

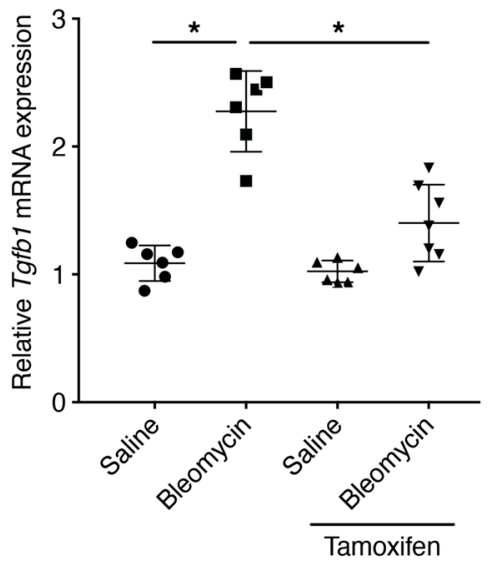

Figure 8. Fibroblast-specific deletion of FOXM1 in a therapeutic protocol protects mice from bleomycininduced fibrosis. (A) Schematic illustrating the timelines for in vivo administration of bleomycin and delayed administration of tamoxifen and determination of experimental end points in the bleomycin model of pulmonary fibrosis. (B) Digital images of Masson's trichrome staining for collagen deposition (blue) at day 21. Original magnification, $\times 200$. Scale bars: $500 \mu \mathrm{m}$. (C-F) Effect of delayed fibroblastspecific FOXM1 deletion in mice treated with and without bleomycin, as reflected by changes in the Ashcroft histology score (C), lung hydroxyproline content (D), and the expression of fibrotic markers Acta2 (E) and Tgfb1 (F) mRNA. In C-F, each symbol represents an individual mouse. Values in each group represent results from 2 pooled independent experiments with a total of 5 to 8 mice per group. ${ }^{*} P<0.05$, 2-way ANOVA with Tukey's multiple comparisons test. broblasts. These cells are marked by their expression of contractile proteins such as $\alpha$-SMA and can be differentiated from resident fibroblasts by treatment with the profibrotic substance TGF- $\beta$. Pretreatment of CCL 210 cells with Sio A $(2.5 \mu \mathrm{M})$ markedly prevented their TGF- $\beta$-induced differentiation into myofibroblasts (reflected by expression and organization into stress fibers of $\alpha$-SMA) as well as expression of COL1A1 (encoding collagen type $1 \alpha 1$ ) (Figure 6, A-C). Pretreatment of fibroblasts with FOXM1 siRNA similarly blunted the induction of ACTA2 (encoding $\alpha$-SMA) in response to TGF- $\beta$ stimulation (Supplemental Figure 6A). Not unexpectedly, IPF fibroblasts exhibited higher basal expression of ACTA2 mRNA than did nonfibrotic fibroblasts (Figure 6D). Of note, both 
A
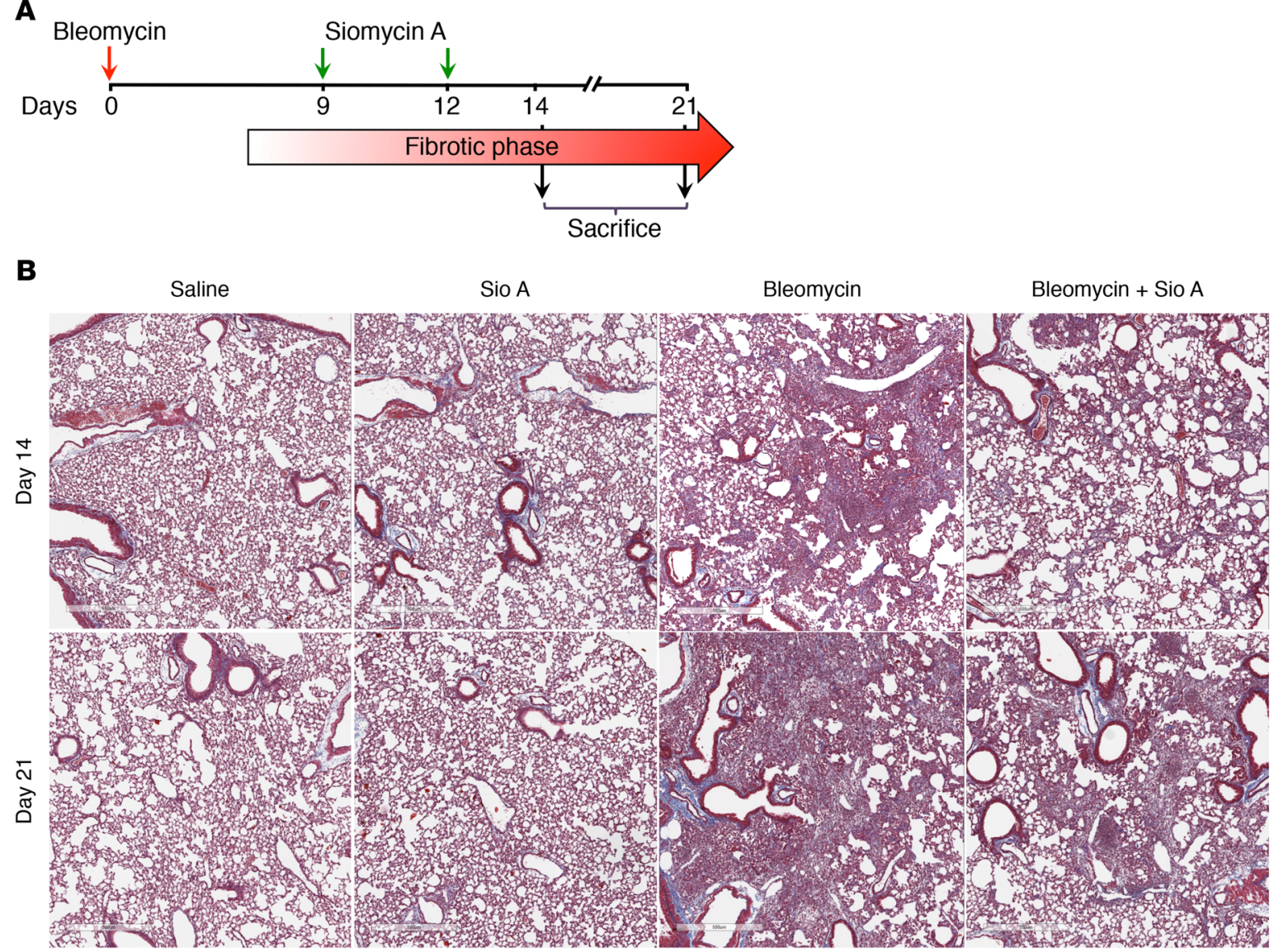

C
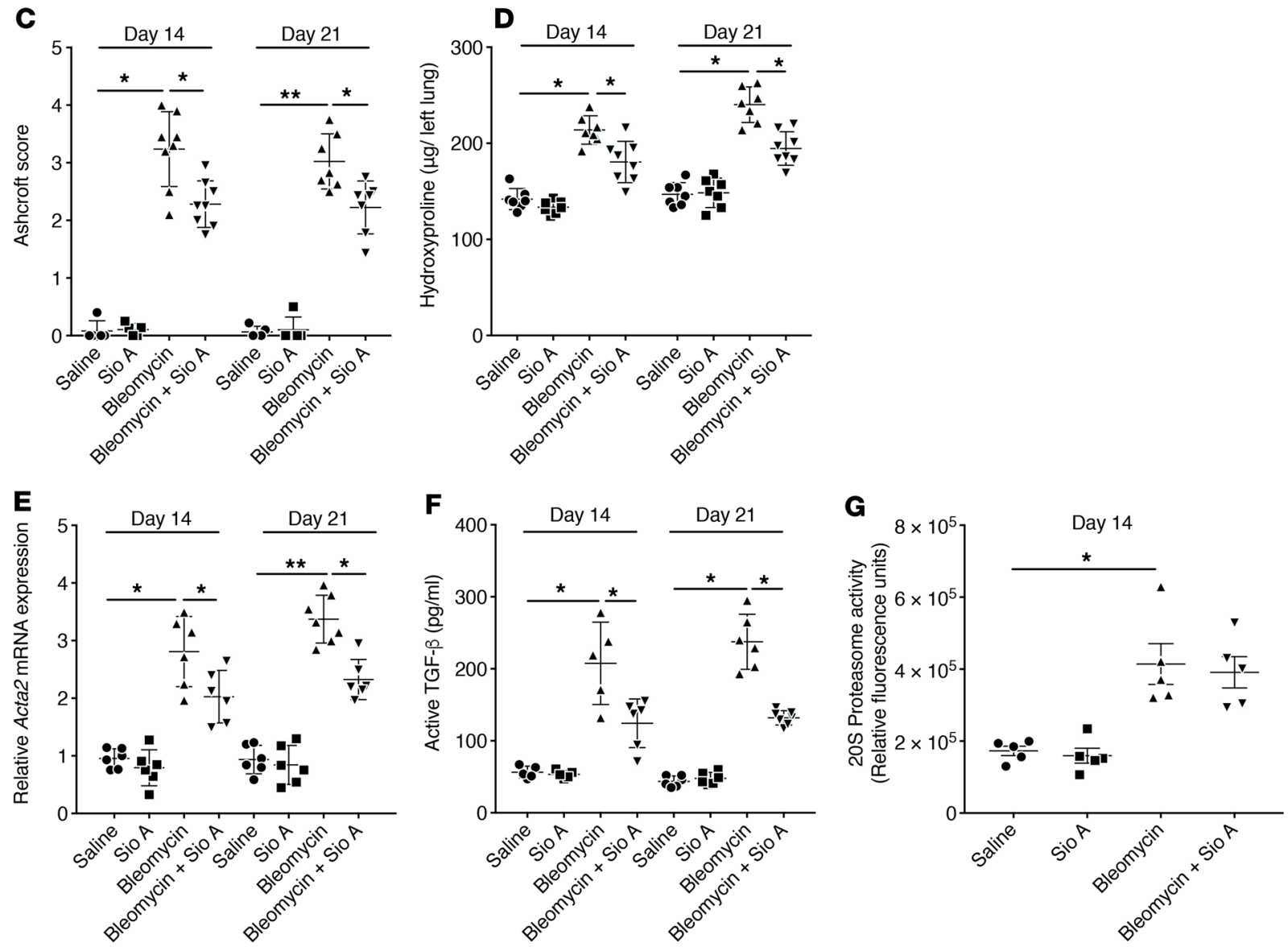
Figure 9. Sio A treatment in a therapeutic protocol ameliorates bleomycininduced fibrosis in mice. (A) Schematic illustrating the timelines for in vivo administration of bleomycin and Sio A, for determination of experimental end points, and for the pertinent phases of the pulmonary response in the bleomycin model of pulmonary fibrosis. (B) Digital images of Masson's trichrome staining for collagen deposition (blue) at days 14 (upper panels) and 21 (lower panels) in mice treated with and without bleomycin and Sio A. Original magnification, $\times 200$. Scale bars: $500 \mu \mathrm{m}$. (C-F) Effect of Sio A treatment in mice treated with and without bleomycin, as reflected by values determined at days 14 and 21 for the Ashcroft histology score (C), lung hydroxyproline content (D), mRNA expression of the myofibroblast marke Acta2 (E), and levels of active TCF- $\beta$ (F). (C) 205 proteasome activity in lung homogenates harvested at day 14 from mice treated with and without bleomycin and with and without Sio A. In C-G, each symbol represents an individual mouse. Values in each group represent results from 2 pooled independent experiments with a total of 5 to 8 mice per group. ${ }^{*} P<0.05$; ${ }^{*} P<0.01,2$-way ANOVA with Tukey's multiple comparisons test.

FOXM1 siRNA and Sio A were able to reduce basal as well as TGF- $\beta$-induced ACTA2 mRNA expression in normal and IPF lines (Figure 6E). Finally, Sio A treatment for 24 hours was also capable of significant reversal of $\alpha$-SMA expression in myofibroblasts that had been differentiated from fibroblasts by prior treatment with TGF- $\beta$ for 24 hours (Figure 6F and Supplemental Figure 6B). These findings suggest a requirement of FOXM1 for myofibroblast differentiation. Although expression levels of FOXM1 and ACTA2 were positively correlated in the entire group of patient-derived fibroblast lines, this relationship did not reach significance in our limited sample (Supplemental Figure 3B).

We next investigated the ability of inhibition of FOXM1 via FOXM1 siRNA or Sio A to promote apoptosis in myofibroblasts - which are notoriously apoptosis resistant $(31,32)$. Apoptosis was determined by Western blot analysis of active (cleaved) caspase 3 (CASP3) and cleaved poly (ADP-ribose) polymerase (PARP), flow cytometric detection of annexin $\mathrm{V}$ staining, and morphologic assessment. In parallel, we also measured protein (by Western blot) and mRNA (by qPCR) levels of expression of apoptosis-related genes. siRNA-mediated knockdown of FOXM1 sensitized the myofibroblasts to Fas ligand-induced (FasL-induced) apoptosis (Figure 6G); in parallel, it increased the expression of proapoptotic genes FAS, CASP3, CASP8, $A P A F 1, B I D$, and $B A D$, while it suppressed the expression of antiapoptotic BIRC5 (Supplemental Figure 6, C-E, and Figure $6 G)$. Likewise, Sio A also markedly sensitized the myofibroblasts to FasL-induced apoptosis (Figure 6, H and I, and Supplemental Figure 6F). Moreover, Sio A by itself was sufficient to induce a modest but significant degree of apoptosis in myofibroblasts (Figure 6, H and I, and Supplemental Figure 6F). In parallel, and consistent with FOXM1 siRNA data, Sio A treatment also increased the expression of FAS and reduced expression of antiapoptotic BIRC5 (Supplemental Figure 6G and Figure 6J). We found a significant positive relationship between FOXM1 expression and BIRC5 expression in the entire group of patientderived fibroblast lines (Supplemental Figure 3C). Taken together, the results shown in Figure 6 and Supplemental Figure 6 demonstrate a previously unrecognized role for FOXM1 in both differentiation and apoptosis resistance of myofibroblasts. Notably, however, this essential role of FOXM1 in myofibroblast maintenance is independent of any direct induction of FOXM1 by TGF- $\beta$ (Supplemental Figure $2 \mathrm{G}$ ).

Deletion of FOXM1 in activated fibroblasts protects from bleomycin-induced fibrosis. Since our in vitro findings provide evidence for upregulated expression of and pleiotropic phenotypic roles for FOXM1 in fibrotic fibroblasts, we next sought to determine the importance of fibroblast FOXM1 in fibrogenesis in vivo. To do so, we engineered mice harboring a fibroblast-specific conditional deletion of FOXM1. We verified that Cre was induced and FOXM1 was deleted in 4-hydroxy tamoxifen-treated lung fibroblasts isolated from these mice (Supplemental Figure 7, A and B). In addition, these fibroblasts exhibited a reduction in Acta2 expression in response to TGF- $\beta$ (Supplemental Figure 7C), consistent with our prior observations with FOXM1 siRNA and Sio A treatment of normal fibroblasts (Figure 6A and Supplemental Figure 6A). We next evaluated these mice in a well-characterized model of bleomycininduced pulmonary fibrosis. As shown in Figure 7A, tamoxifen was administered i.p. 3 days prior to the single oropharyngeal dose of bleomycin (on day 0) and every 3 days thereafter from day 0 to day 18 to both FOXM1/f/fl Col1a2-Cre-ER(T) ${ }^{+/ 0}$ (fibroblast-specific knockout) and Colla2-Cre-ER(T) $+/ 0$ (control) mice. Tamoxifeninduced expression of Cre in the lungs of FOXM1 $1^{t / f l}$ Colla2 mice was confirmed by qPCR (Supplemental Figure 7D). As expected, bleomycin administration to control mice resulted in infiltration and obliteration of alveolar spaces, as evident from morphologic evaluation (Figure 7B) and reflected in the increased Ashcroft score (Figure 7C). It also elicited a significant increase in lung hydroxyproline content (Figure 7D), a measure of collagen deposition. Conditional fibroblast-specific FOXM1-knockout mice showed no changes in lung morphology and integrity when given saline. However, after bleomycin administration, these mice showed a significantly reduced degree of alveolar infiltration (Figure 7B) and obliteration, as measured by the Ashcroft score (Figure 7C). They also exhibited a significant reduction in collagen deposition, as reflected by hydroxyproline measurement (Figure 7D). Furthermore, lung tissue from the fibroblast-specific FOXM1-knockout mice demonstrated a significant reduction in expression of mRNA encoding the fibrotic markers Acta2, Col1a1, Tgfb1, and connective tissue growth factor (Ctgf) (Figure 7, E-H).

The bleomycin model is well recognized as involving acute inflammation and lung injury within the first few days following bleomycin administration, which wanes by days 7 through 10 and is followed by a fibrotic phase evolving over days 14 through 28 . In order for this model to have translational applicability to the treatment of patients who already manifest fibrotic lung disease on clinical presentation, it is necessary to test the efficacy of intervention applied exclusively during the fibrotic phase (33). Therefore, rather than tamoxifen administration beginning 3 days prior to bleomycin administration (Figure 7), we also tested the effect of delaying the initiation of tamoxifen administration to day 9 after bleomycin administration, with additional doses every 3 days from day 12 to day 18 to both FOXM1 ${ }^{f l / l}$ Colla2-Cre-ER(T) ${ }^{+/ 0}$ (fibroblastspecific knockout) and Col1a2-Cre-ER(T) ${ }^{+/ 0}$ (control) mice (Figure 8A). Delayed administration of tamoxifen to bleomycin-treated conditional fibroblast-specific FOXM1-knockout mice resulted in a significant reduction in alveolar infiltration (Figure 8B), alveolar obliteration as measured by the Ashcroft score (Figure $8 \mathrm{C}$ ), and 
collagen deposition as reflected by hydroxyproline measurement (Figure 8D). Indeed, the degree of abrogation of these indices of bleomycin-induced fibrosis was comparable to that afforded by initiation of tamoxifen in the pretreatment protocol (Figure 7). Lung tissue from these mice also demonstrated a significant reduction in expression of mRNA encoding the fibrotic markers Acta 2 and $T g f b 1$ (Figure 8, E and F). Collectively, these data demonstrate that FOXM1 in fibroblasts is necessary for the development of lung fibrosis and that even delaying its deletion from fibroblasts to the fibrotic phase is sufficient to result in marked protection from fibrosis.

In vivo treatment with Sio A protects mice from bleomycin-induced fibrosis. To translate the above findings to a more tractable therapeutic context, we asked whether pharmacologic inhibition of FOXM1 with Sio A was able to ameliorate bleomycin-induced pulmonary fibrosis in WT C57BL/6 mice. To preserve translational utility as discussed above, we limited administration of Sio A to the early fibrotic phase (days 9 and 12) (Figure 9A). The dose of Sio A utilized was based on unpublished murine toxicity data available at the NCI Developmental Therapeutics Program database (https:// dtp.cancer.gov/dtpstandard/servlet/InvivoScreen?testshortna me $=$ Tumor + B1 $+\% 28$ ip $\% 29+$ in $+02 \&$ searchtype $=$ NSC\&searchl ist $=285116$ ) and on published antitumor effects of a related compound (34). As expected, and as previously demonstrated in Figures 7 and 8, bleomycin administration resulted in infiltration and obliteration of alveolar spaces (Figure 9B) reflected in the increased Ashcroft score (Figure 9C) as well as a significant increase in lung hydroxyproline content (Figure 9D), indicative of exaggerated collagen deposition. Treatment with Sio A alone had no effect on any of the measured end points. However, delayed treatment with Sio A significantly attenuated the alveolar infiltration/ obliteration (Figure 9, B and C) and the increment in lung hydroxyproline (Figure 9D) and Acta2 mRNA (Figure 9E) in bleomycininjured mice assessed at both day 14 and day 21. In addition, the increase in active TGF- $\beta$ detected in bronchoalveolar lavage (BAL) fluid of bleomycin-injured mice on days 14 and 21 was significantly attenuated with Sio A treatment (Figure 9F). Since Sio A has been reported to cause apoptosis in malignant epithelial cells (22), we evaluated its effects at a concentration of $2.5 \mu \mathrm{M}$ on apoptosis in murine lung epithelial cells (MLE-12). As shown in Supplemental Figure 8A, treatment with Sio A alone was not sufficient to elicit generation of cleaved PARP. Moreover, Sio A failed to potentiate the effect of FasL on PARP cleavage. Thus, Sio A at this concentration lacked the enhancing effects on apoptosis in epithelial cells that it exerted in fibroblasts (Figure 6 and Supplemental Figure 6). Next, we assessed the effects of Sio A on alveolar cell apoptosis in vivo using IHC staining for CASP3 on sections of lungs harvested on day 14 from control or Sio A-treated mice following saline or bleomycin challenge. As shown in Supplemental Figure 8B, Sio A administration did not increase the number of CASP3-positive cells. Because of the potential of Sio A to inhibit the proteasome (19), we also measured proteasome activity in lung homogenates harvested at day 14 from control or Sio A-treated mice following saline or bleomycin challenge. As shown in Figure 9G, bleomycin increased lung proteasome activity, as has been reported by others (35). However, Sio A administration had no effect on proteasome activity in the lungs of either saline-treated or bleomycin-injured mice. These results demonstrate that global pharmacologic inhibition of FOXM1 with Sio A during the fibrotic phase of response was able to attenuate bleomycin-induced pulmonary fibrosis without causing widespread apoptosis of alveolar cells or detectable proteasome inhibition.

\section{Discussion}

Information about the possible role of FOXM1 in tissue fibrogenesis is limited and conflicting. Its postnatal deletion from cardiomyocytes led to cardiac fibrosis (36), while on the other hand, its deletion from alveolar epithelial cells protected against radiationinduced pulmonary fibrosis and epithelial-mesenchymal transition (EMT) (37). Recent studies using lineage tracing to explore the origins of myofibroblasts - the ultimate effectors of tissue fibrosis - in models of lung fibrosis have concluded that an in vivo role for EMT is either absent or minimal (38-40) and instead emphasize the importance of resident lung fibroblasts as their major source $(39,40)$. Despite this, FOXM1 has never previously been interrogated in fibroblasts. However, since FOXM1 has been most extensively studied in cancer, it is particularly interesting that numerous parallels in phenotype between fibrotic fibroblasts and cancer cells have long been appreciated (41). We found that baseline FOXM1 expression was low in normal lung fibroblasts, but was increased in response to mitogens and was also constitutively higher in cells isolated from the fibrotic lungs of both humans with IPF and mice challenged with bleomycin. Parallel results using IHC staining of lung sections revealed increased FOXM1-positive cells within the interstitium of fibrotic regions of both human IPF patients and bleomycin-treated mice. The accumulation of mesenchymal cells in fibrotic tissue reflects a combination of proliferation and apoptosis resistance (42). The differentiation of fibroblasts to myofibroblasts is also critical, as both the expression of contractile proteins and the augmented elaboration of collagen by these cells contribute to the tissue stiffness that leads to physiologic organ impairment (43). Using both molecular and pharmacologic approaches to interrogate the functional roles of FOXM1, we found that it has the potential to contribute to each of these pivotal phenotypic features of fibrotic fibroblasts.

Both pharmacologic inhibition and knockdown of FOXM1 prevented proliferation as well as induction of cell cycle-regulated genes in response to various mitogens. While FOXM1 is well recognized as a driver of the cell cycle based on studies in other cell types, predominantly cancer cells, our data are the first, to our knowledge, to establish its importance in driving proliferation of human lung fibroblasts. Indeed, a strong positive correlation between FOXM1 expression and proliferative capacity was observed in patient-derived fibroblasts, with IPF cells expressing higher levels of both parameters than nonfibrotic control cells. Inhibition of FOXM1 by both molecular and pharmacologic approaches similarly inhibited TGF- $\beta$-induced myofibroblast differentiation. Sio A was also able to reverse (i.e., dedifferentiate) the phenotype of established myofibroblasts differentiated by TGF- $\beta$ pretreatment. In cells isolated from the lungs of IPF patients - which at baseline (in the absence of exogenous TGF- $\beta$ ) exhibit the increased $\alpha$-SMA expression typical of myofibroblasts - both FOXM1 knockdown and inhibition with Sio A likewise promoted dedifferentiation, as reflected by attenuated expression of this contractile protein. Our 
data linking myofibroblast differentiation with active FOXM1 is consistent with another report linking such differentiation with low levels of FOXO3A (28), a known competitor of FOXM1. However, the mechanism by which FOXM1 promotes this differentiated phenotype remains to be determined.

Whereas we found FOXM1 to be both necessary and sufficient for proliferation, it was necessary but not sufficient for differentiation. Although fibroblast proliferation and differentiation have been suggested as representing mutually exclusive cellular programs, this may represent an overly simplistic conceptualization. For example, serum-response factor, a transcription factor that has been shown to be instrumental in myofibroblast differentiation $(44,45)$, has also been reported as regulating fibroblast proliferation $(46,47)$. It is thus possible that these phenotypic programs are in fact interdependent and that ultimate-fate decisions depend on contextual cues that remain to be fully elucidated. It is also possible that these distinct cell programs operate within individual clones or subsets within the mixed populations of fibroblasts that grow out of lung explants. The possible role of FOXM1 in such cellfate decisions will be of interest in future investigations.

Increased expression of BIRC5 and decreased expression of FAS have been suggested as contributing to the apoptosis resistance of IPF fibroblasts $(31,48)$. It was therefore of interest that, in parallel with increasing susceptibility to FasL-induced apoptosis, FOXM1 knockdown caused downregulation of BIRC5 and upregulation of FAS as well as a number of other proapoptotic genes (CASP3, CASP8, APAF1, BID, and BAD). Our results thus suggest that the increased expression of FOXM1 in IPF fibroblasts may contribute to the well-recognized apoptosis resistance of these cells. As the lungs of patients with IPF manifest accumulation of pathogenic myofibroblasts at the time of clinical presentation, the potential of pharmacologic agents targeting FOXM1 to promote both their apoptosis and their dedifferentiation constitutes a highly desirable feature. Approximately one-third of the fibroblast lines obtained from individual IPF patients in our sample failed to manifest an upregulation of FOXM1 protein expression, consistent with the well-recognized phenomenon of molecular heterogeneity among patient-derived cell lines in this disease (11, 12) and the likely need to personalize therapeutic targeting of this transcription factor in individual patients.

No biological mediator serving as a brake on FOXM1 expression/ activation has been recognized to date, but we showed that the lipid autocoid $\mathrm{PGE}_{2}$ acts as an endogenous brake on FOXM1. Although the biological actions of $\mathrm{PGE}_{2}$ are highly pleiotropic and context dependent, its effects on fibroblasts are largely suppressive and include inhibition of proliferation (49) and survival (50) as well as both inhibition and reversal of established myofibroblast differentiation (51). All of these actions are mediated by cAMP signaling downstream of the EP2 receptor, and the effects of a selective EP2 agonist (butaprost) as well as a substance that bypasses EP2 to directly activate cAMP synthesis (forskolin) implicate this same signaling pathway in the inhibition of FOXM1 activation by $\mathrm{PGE}_{2}$. Since cAMP also mediates the actions of numerous other $\mathrm{G}$ protein-coupled receptor agonists and pharmacologic agents (including prostacyclin, adenosine, adrenomedullin, and $\beta$-adrenergic agonists), such modulation of FOXM1 activation may extend to a variety of other biologically relevant situations. $\mathrm{PGE}_{2}$ has previously been shown to dephosphorylate AKT
(27) and abolish PI3K signaling in fibroblasts, and we confirmed that this results in the dephosphorylation and nuclear accumulation of the FOXM1 competitor FOXO3A. However, the fact that $\mathrm{PGE}_{2}$ inhibition of FOXM1 persisted to a substantial degree in the presence of Myr-AKT as well as FOXO3A knockdown suggests an additional FOXO3A-independent mechanism by which $\mathrm{PGE}_{2}$ interferes with FOXM1 binding to target gene-promoter elements. One possible mechanism that merits future exploration is induction of $\mathrm{P} 21$, originally described as a cyclin-dependent kinase inhibitor, but now also recognized as interfering with FOXM1 binding (52), which has been reported to be induced by forskolin (53). PGE $_{2}$ inhibition of fibroblast expression/activation of FOXM1 thus represents yet an additional mechanism for the antifibrotic actions of this prostanoid. We and others have previously shown that interfering with $\mathrm{PGE}_{2}$ synthesis $(54,55)$ or its actions via EP2 (26) worsens bleomycin-induced fibrosis, and it is certainly possible that such potentiation of fibrosis reflects the loss of this brake on FOXM1. We did not attempt to verify $\mathrm{PGE}_{2}$ regulation of FOXM1 in vivo, as the downregulation of the operative EP2 receptor in fibrotic lung fibroblasts $(26,56)$ would interfere with the ability of $\mathrm{PGE}_{2}$ administration to suppress (owing to the cells' $\mathrm{PGE}_{2}$ resistance) FOXM1. As fibrosis is already a state of $\mathrm{PGE}_{2}$ deprivation, further ablating $\mathrm{PGE}_{2}$ synthesis or signaling would have little effect on potentiating FOXM1.

We sought to evaluate the contribution of FOXM1 to in vivo fibrogenesis by selectively deleting it from Col1-expressing cells. Under baseline conditions, Col1a2 expression in the lung is almost entirely limited to resident fibroblasts (57). It is well known that during fibrotic responses, other cells, including epithelial cells, can undergo mesenchymal transition $(39,58)$, thus expressing Col1 and being subject to Cre-mediated deletion under the control of the Col1 promoter. Although this is a limitation of the specificity of a Col1a2-driven Cre system to delete FOXM1 from fibroblasts, no gene is currently recognized as being superior to Col1 for its ability to specifically distinguish fibroblasts from other cell types in a fibrotic milieu. Furthermore, lineagetracing studies strongly suggest that resident fibroblasts are the predominant source of Col1 expression and of myofibroblasts in bleomycin-induced lung fibrosis (40). Therefore, we utilized inducible Col1a2 promoter-driven expression of Cre recombinase in FOXM1 $1^{f / f l}$ mice (conditional FOXM1-knockout mice) and suggest that use of the Col1a2-driven Cre system represents the best available approach to understanding the contribution of a fibroblast-specific gene. In both prevention and treatment protocols for conditional deletion of FOXM1, we found no basal changes in lung morphology or extracellular matrix deposition. However, the architectural distortion and increase in extracellular matrix deposition elicited by bleomycin administration was markedly attenuated in conditional FOXM1-knockout mice using both protocols. Parallel decreases in gene expression of Col1a1 and Acta2 accompanied the amelioration of fibrosis. These studies provide evidence of the importance of fibroblast FOXM1 in organ fibrosis in vivo and are entirely consistent with the ability of this transcription factor to control a variety of profibrotic functional properties of these cells in vitro. Although we have herein demonstrated substantive roles for FOXM1 in proliferation, survival, and differentiation of fibroblasts, the relative importance of inhibition of these processes in the pro- 
tection against bleomycin-induced lung fibrosis observed in fibroblast-specific conditional FOXM1-knockout mice requires further investigation.

Sio A is a thiazole antibiotic shown to inhibit the transcriptional activity and expression of FOXM1 by interference with its DNA binding (59). Although its in vitro efficacy has been amply documented (60), there are no prior published reports of its systemic administration. We tested its antifibrotic potential in the bleomycin fibrosis model by dosing it exclusively during the early fibrotic phase of response. Such a "therapeutic" protocol is more clinically relevant to treatment of patients with established IPF, who by definition lack an antecedent history of symptomatic lung injury. Administering only 2 doses of Sio A was sufficient to provide statistically significant $(P<0.05)$ protection in all the fibrotic end points measured. We also excluded proteasome inhibition in the lungs of these treated mice. To our knowledge, these data represent the first demonstration of the in vivo efficacy of Sio A in any disease model. The degree of protection with Sio A, however, was less than that observed in the fibroblast-specific FOXM1-knockout mice. We speculate that greater in vivo efficacy of Sio A might have been realized with more intensive dosing regimens than that utilized here. Optimal dosing and route of administration remain to be defined. Furthermore, a number of classes of small molecule inhibitors of FOXM1 are currently under development $(61,62)$, and these may be more attractive therapeutic candidates for treatment of fibrotic lung disease than is Sio A.

Although the protection from fibrosis observed in mice harboring a Col1a2 promoter-driven deletion of FOXM1 argues strongly for a critical role for this transcription factor in fibroblasts, the protection provided by in vivo administration of Sio A could have reflected its actions in other cell types as well. The possibility that protection from EMT (37) may have contributed to the therapeutic effect of Sio A cannot, of course, be excluded. Another potential cellular target is macrophages. The critical profibrotic mediator TGF- $\beta$ can derive from a variety of cell types, including fibroblasts themselves (63-66). Our data with fibroblast-specific FOXM1-knockout mice implicate a role for the fibroblast in the generation of this mediator, along with the TGF- $\beta$ target gene product CTGF. However, these data do not distinguish between a direct and an indirect role (via macrophages) for the fibroblast in TGF- $\beta$ production. In this regard, FOXM1 has been implicated in both recruitment and functional modulation of macrophages in a butylated hydroxytoluene-induced model of lung inflammation (13). Since M2 macrophages promote tissue fibrosis in part via elaboration of TGF- $\beta$ (67), it is possible that the inhibitory effects of Sio A on lung TGF- $\beta$ levels may also reflect FOXM1 targeting of macrophages.

Overall, our findings identify FOXM1 as a newly identified driver of the activation state of fibrotic fibroblasts and a therapeutic target for the treatment of fibrotic diseases of the lung and, potentially, of other organs. The fact that FOXM1 integrates the actions of not only mitogens, but also of TGF- $\beta$, suggests that it may offer advantages as a therapeutic target over exclusively targeting mitogen receptors with an agent such as nintedanib.

\section{Methods}

Cells, tissue culture, and reagents. CCL210 fibroblasts (CCD-19Lu), a commercially available primary line of fibroblasts isolated from normal adult human lung, the immortalized normal human bronchial epithelial cell line, BEAS-2B, and MLE-12 cells, a murine lung epithelial cell line, were purchased from ATCC. Selected studies were also performed with lung fibroblasts isolated at our institution under an IRB-approved protocol described previously (25) from lung tissue determined histologically to be either nonfibrotic or diagnostic of IPF. Fibroblasts were also isolated from the lungs of saline- or bleomycin-treated mice as described previously (68). Fibroblast lines used in this study were all at passage numbers 8-10. Both fibroblasts and epithelial cells were cultured in DMEM (Invitrogen) supplemented with $10 \%$ fetal bovine serum (Hyclone) in a $\mathrm{CO}_{2}$ incubator at $37^{\circ} \mathrm{C}$. Recombinant human TGF- $\beta$, FGF2, and PDGF were purchased from R\&D Systems. Sio A was obtained from the Developmental Therapeutics Program of the National Cancer Institute (http:// dtp.nci.nih.gov). Pharmacologic agents $\mathrm{PGE}_{2}$, forskolin, butaprost, U0126, PD98059, LY294002, and triciribine were purchased from Cayman Chemicals. GSK 2334470 was purchased from Tocris Bioscience. Unless otherwise specified, Sio A was used at $2.5 \mu \mathrm{M}$, TGF- $\beta$ was used at $2 \mathrm{ng} / \mathrm{ml}, \mathrm{FGF} 2$ and PDGF were each used at $25 \mathrm{ng} / \mathrm{ml}$, and $\mathrm{PGE}_{2}$ was used at a final concentration of $0.5 \mu \mathrm{M}$.

Mice. WT female C57BL/6 mice were obtained from Charles River Laboratories and used at 8 weeks of age. Transgenic conditional FOXM1knockout mice of both sexes were bred as follows on a C57BL/6 background. Transgenic Colla2-Cre-ER(T $)^{+/ 0}$ mice and FOXM1 $1^{t / f l}$ mice were obtained from Sem Phan (University of Michigan) (69) and Vladimir Kalinichenko (Cincinnati Children's Hospital, Cincinnati, Ohio, USA), respectively (70). To generate fibroblast-specific FOXM1-deficient mice, FOXM1 $1^{f / f l}$ mice were bred with Colla2-Cre-ER(T $)^{+/ 0}$ (heterozygous allele) transgenic mice to generate mice heterozygous for both alleles. Progeny from the second cross between FOXM1 $1^{f / f l}$ mice and heterozygous FOXM1 $1^{f / W T}$ Colla2-Cre-ER(T) ${ }^{+/ 0}$ mice (from the first cross) were used for further experiments. All mice were genotyped by PCR techniques as described previously $(69,70)$. For treatment of mice, a stock solution of tamoxifen (Sigma-Aldrich) in ethanol $(100 \mathrm{mg} / \mathrm{ml})$ was diluted in corn oil to $10 \mathrm{mg} / \mathrm{ml}$ (71). To selectively delete FOXM1 in activated fibroblasts, adult $F O X M 1^{f / f l}$ Colla2-Cre-ER $(T)^{+/ 0}$ mice (8-10 weeks old) and the relevant control (Col1a2-Cre-ER(T) ${ }^{+/ 0}$ with WT FOXM1) mice were administered tamoxifen suspension ( $0.1 \mathrm{ml}$ of diluted stock) via i.p. injection, beginning either at 3 days before or 9 days after administration of bleomycin and every 72 hours thereafter until sacrifice.

Plasmid transfection. The FOXM1 overexpression plasmid CMV T7-FOXM1 was provided by Pradip Raychaudhuri (University of Illinois at Chicago, Chicago, Illinois, USA) and a Myr-AKT construct was a gift of Philip Tsichlis (Tufts University, Boston, Massachusetts, USA) $(16,24)$. Transient transfection studies were performed with overexpression or control plasmids using FuGENE HD (Promega). In brief, plasmid and transfection reagents were mixed at a 1:3 DNA/ FuGENE HD ratio, incubated for 15 minutes at room temperature, and added to the cells. Twenty-four hours after transfection, cells were cultured in serum-free medium for another 24 hours before treating cells with and without $\mathrm{PGE}_{2}$. Samples were harvested per the experimental protocol.

Western blot analysis. Cells were lysed in RIPA buffer supplemented with protease inhibitors (Roche Diagnostics) and phosphatase inhibitor cocktail (EMD Biosciences). Where applicable, nuclear and cytoplasmic fractions were separated using the NE-PER Nuclear and Cytoplasmic Extraction Kit (Thermo Scientific). Proteins were separated by SDSPAGE and transferred to nitrocellulose membrane. Membranes were 
blocked with $5 \%$ nonfat dry milk and were probed with primary antibodies against FOXM1 (catalog ABN286, Millipore); CCND1 (catalog sc-8396) and Sam 68 (catalog sc-514468) purchased from Santa Cruz Biotechnology; CCNB1 (catalog 4135), PLK1 (catalog 4513), AKT (catalog 9272), p-AKT (catalog 4051), ERK1/2 (catalog 9102), p-ERK1/2 (catalog 9106), FOXO3A (catalog 12829), FAS (catalog 4233), CASP3 (catalog 9665), cleaved PARP (catalog 5625), and GAPDH (catalog 3683) purchased from Cell Signaling Technologies; $\alpha$-SMA (catalog ab7817), pFOXO3A (catalog ab154786), and BIRC5 (catalog ab76424) purchased from Abcam; collagen 1A1 (catalog PA5-29569) purchased from Thermo Scientific; and $\alpha$-tubulin (catalog T9026; clone DM1A) purchased from Sigma-Aldrich. All antibodies were used at a dilution of 1:1,000 unless specified otherwise. Full, uncut gels for all the Western blots are shown in the supplemental material.

Fibroblast proliferation. Proliferation studies in lung fibroblasts were performed using the CyQUANT NF Cell Proliferation Assay Kit (Thermo Fisher). Briefly, fibroblasts in complete medium (DMEM with $10 \% \mathrm{FBS}$ ) were plated at $5 \times 10^{3}$ cells/well in a 96-well plate, and after 24 hours, cells were serum starved for 16 hours. Cells were then treated with and without FGF2, PDGF, or TGF- $\beta$ in serum-free DMEM for 72 hours at $37^{\circ} \mathrm{C}$. Cells were washed with PBS and frozen at $-20^{\circ} \mathrm{C}$. To study the effects of inhibitors on lung fibroblast proliferation, cells were pretreated with and without Sio A $(2.5 \mu \mathrm{M}), \mathrm{PGE}_{2}(0.5 \mu \mathrm{M})$, forskolin $(10 \mu \mathrm{M})$, or butaprost $(10 \mu \mathrm{M})$ for 30 minutes prior to stimulation with FGF2. Cells were thawed, and $100 \mu$ l of lysis buffer containing the CyQUANT GR dye was added to each well and incubated at $37^{\circ} \mathrm{C}$ for 30 minutes. Fluorescence was measured using a fluorescence microplate reader with excitation of $485 \mathrm{~nm}$ and emission of $530 \mathrm{~nm}$. All experiments were performed in triplicate and the results presented as mean \pm SEM of at least 3 independent experiments.

Fibroblast differentiation. Myofibroblast differentiation was achieved by stimulating the fibroblasts with TGF- $\beta$ for 48 hours. Myofibroblasts were identified by increased expression of ACTA2 and COL1A1, as measured by qPCR and Western blot, and by immunofluorescence microscopic visualization of $\alpha$-SMA stress fibers.

Fibroblast apoptosis. Fibroblast apoptosis was evaluated at baseline and after treatment with FasL. Apoptosis was determined by measuring cell-surface expression of phosphatidylserine using annexin V-FITC staining and analyzed by flow cytometry $(72,73)$. Cleaved (active) CASP3 and cleaved PARP were analyzed by Western blot. Morphological alterations characteristic of apoptosis, such as cell rounding, cytoplasmic shrinking, and detachment (74), were documented by phase-contrast microscopy.

Proteasome activity assay. Cells were seeded at $1 \times 10^{4}$ cells/ well in a 96-well plate in complete medium (DMEM with 10\% FBS) and incubated for 24 hours. Cells were then treated with different doses of Sio A or MG132 or an equal volume of DMSO in triplicate. After 24 hours of incubation, proteasome activity was quantified in cell lysates using the 20S Proteasome Activity Assay Kit, purchased from Cayman Chemicals, with the substrate Suc-LLVY-AMC. Likewise, proteasome activity was quantified using this same assay in whole-lung tissue homogenates from bleomycin-injured mice treated with and without Sio A or saline.

Bleomycin model of pulmonary fibrosis. Fibrosis was elicited in mice by administration of a single oropharyngeal dose of 1.5 units $/ \mathrm{kg}$ body weight of bleomycin (MilliporeSigma); control mice received a volume of sterile saline equal to that described previously (75). Mice were sac- rificed at the indicated times. BAL fluids were collected to determine the levels of active TGF- $\beta$ in lavage fluid by ELISA (described below), and the left lung was analyzed for hydroxyproline (76), while the right lung lobes were assessed for expression of fibrotic marker mRNAs (Acta2, Col1a1, Tgfb1, and Ctgf) and histopathology. Histopathologic analysis is described below.

Sio A administration. At designated time points (day 9 and day 12) after bleomycin administration, the FOXM1 inhibitor Sio A or saline was administered via the i.p. route at a dose of $25 \mathrm{mg} / \mathrm{kg}$ body weight. We utilized 6 to 8 mice per treatment group for each time point.

Histopathology and Ashcroft score. Lungs were fixed in $10 \%$ neutralbuffered formalin and embedded in paraffin. Sections $5-\mu \mathrm{m}$ thick were stained with Masson's trichrome to detect collagen and visualize the extent of fibrosis. Whole slide images were generated from digitally scanned slides using commercial image analysis software (Leica Aperio Positive Pixel Count algorithm, v9). The validated semiquantitative Ashcroft score was used to score pulmonary fibrosis in the digitized sections $(77,78)$. In brief, using $\times 20$ magnification, each of 10 successive fields was given a score ranging from 0 (normal lung) to 8 (total fibrous obliteration of the field). The mean score of all 10 fields was taken as the fibrosis score.

Additional methods, including FOXM1 adenovirus transduction, quantitative reverse-transcriptase PCR, siRNA transfection, quantitative ChIP assay, immunofluorescence microscopy, IHC, and TGF- $\beta$ ELISA are described in the Supplemental Methods. The primer sequences used for quantitative reverse-transcriptase PCR and quantitative ChIP assay are listed in Supplemental Table 1. siRNA sequences used for knockdown studies are listed in Supplemental Table 2.

Statistics. Unless specified otherwise, all in vitro data were from a minimum of 3 independent experiments. In vivo data reflect 6 to 8 mice per group. Data are reported as mean \pm SEM and were compared using the unpaired 2-sided Student's $t$ test. When multiple comparisons were necessary, 1-way or 2-way ANOVA with post hoc Bonferroni's correction was used. Relationships between FOXM1 mRNA expression and select phenotypes or genes in nonfibrotic and IPF patient-derived fibroblasts were evaluated using correlation analysis. $P<0.05$ was considered statistically significant.

Study approval. All animal experiments were conducted with the approval of the University of Michigan's IACUC. Because all of the patient samples were deidentified, the University of Michigan IRB deemed these studies exempt from IRB approval.

\section{Author contributions}

LRP planned and performed experiments, analyzed the data, organized data for presentation, and wrote the manuscript. JMS and VLD performed experiments. ILB performed the histopathologic analyses. ESW provided the patient specimens and assistance with their use. MPG planned experiments, analyzed data, and wrote the manuscript.

\section{Acknowledgments}

We thank Sem Phan (University of Michigan) for providing Col1a2-Cre-ER $(T)^{+/ 0}$ mice and Vladimir Kalinichenko (Cincinnati Children's Hospital) for providing FOXM1 $1^{f / f l}$ mice. We thank Pradip Raychaudhuri (University of Illinois at Chicago) for the kind gift of CMV T7-FOXM1 full-length plasmid, Guy Adami (University of Illinois at Chicago) for adenovirus expressing FOXM1, and 
Philip Tsichlis (Tufts University) for the Myr-AKT plasmid. We also thank the National Cancer Institute Developmental Therapeutics Program for providing the Sio A used in our studies. We acknowledge the technical assistance of Hideyasu Ouchi as well as the helpful input from members of the Peters-Golden laboratory. This work was supported by NIH grant HL094311 (to MPG), an
American Heart Association Fellowship Award (to LRP), and NIH Training Grant T32 HL7749-23 (to JMS).

Address correspondence to: Marc Peters-Golden, 6301 MSRB III, 1150 W. Medical Center Drive, Ann Arbor, Michigan 48109, USA. Phone: 734.936.5047; Email: petersm@umich.edu.
1. Wynn TA, Ramalingam TR. Mechanisms of fibrosis: therapeutic translation for fibrotic disease. Nat Med. 2012;18(7):1028-1040.

2. Raghu G, Selman M. Nintedanib and pirfenidone. New antifibrotic treatments indicated for idiopathic pulmonary fibrosis offer hopes and raises questions. Am J Respir Crit Care Med. 2015;191(3):252-254.

3. Thannickal VJ, et al. Matrix biology of idiopathic pulmonary fibrosis: a workshop report of the national heart, lung, and blood institute. Am J Pathol. 2014;184(6):1643-1651.

4. Xue L, Chiang L, He B, Zhao YY, Winoto A. FoxM1, a forkhead transcription factor is a master cell cycle regulator for mouse mature $\mathrm{T}$ cells but not double positive thymocytes. PLOS ONE. 2010;5(2):e9229.

5. Zhang H, et al. The FoxM1 transcription factor is required to maintain pancreatic beta-cell mass. Mol Endocrinol. 2006;20(8):1853-1866.

6. Bella L, Zona S, Nestal de Moraes G, Lam EW. FOXM1: A key oncofoetal transcription factor in health and disease. Semin Cancer Biol. 2014;29:32-39.

7. Koo CY, Muir KW, Lam EW. FOXM1: From cancer initiation to progression and treatment. Biochim Biophys Acta. 2012;1819(1):28-37.

8. Halasi M, Gartel AL. A novel mode of FoxM1 regulation: positive auto-regulatory loop. Cell Cycle. 2009;8(12):1966-1967.

9. Wang IC, et al. Forkhead box M1 regulates the transcriptional network of genes essential for mitotic progression and genes encoding the SCF (Skp2-Cks1) ubiquitin ligase. Mol Cell Biol. 2005;25(24):10875-10894.

10. Barrett RM, Colnaghi R, Wheatley SP. Threonine 48 in the BIR domain of survivin is critical to its mitotic and anti-apoptotic activities and can be phosphorylated by CK2 in vitro. Cell Cycle. 2011;10(3):538-548.

11. Moodley YP, et al. Comparison of the morphological and biochemical changes in normal human lung fibroblasts and fibroblasts derived from lungs of patients with idiopathic pulmonary fibrosis during FasL-induced apoptosis. J Pathol. 2004;202(4):486-495.

12. Phan SH. Biology of fibroblasts and myofibroblasts. Proc Am Thorac Soc. 2008;5(3):334-337.

13. Balli $\mathrm{D}$, et al. Foxm 1 transcription factor is required for macrophage migration during lung inflammation and tumor formation. Oncogene. 2012;31(34):3875-3888.

14. Wollin L, et al. Mode of action of nintedanib in the treatment of idiopathic pulmonary fibrosis. Eur Respir J. 2015;45(5):1434-1445.

15. Conte E, et al. Inhibition of PI3K prevents the proliferation and differentiation of human lung fibroblasts into myofibroblasts: the role of class I P110 isoforms. PLoS ONE. 2011;6(10):e24663.
16. Park HJ, Wang Z, Costa RH, Tyner A, Lau LF, Raychaudhuri P. An N-terminal inhibitory domain modulates activity of FoxM1 during cell cycle. Oncogene. 2008;27(12):1696-1704.

17. Liu Y, et al. FoxM1 mediates the progenitor function of type II epithelial cells in repairing alveolar injury induced by Pseudomonas aeruginosa. JExp Med. 2011;208(7):1473-1484.

18. Gartel AL. Thiazole antibiotics siomycin a and thiostrepton inhibit the transcriptional activity of FOXM1. Front Oncol. 2013;3:150.

19. Bhat UG, Halasi M, Gartel AL. FoxM1 is a general target for proteasome inhibitors. PLOS ONE. 2009;4(8):e6593.

20. Han YH, Moon HJ, You BR, Park WH. The effect of MG132, a proteasome inhibitor on HeLa cells in relation to cell growth, reactive oxygen species and GSH. Oncol Rep. 2009;22(1):215-221.

21. Park WH, Kim SH. MG132, a proteasome inhibitor, induces human pulmonary fibroblast cell death via increasing ROS levels and GSH depletion. Oncol Rep. 2012;27(4):1284-1291.

22. Bhat UG, Halasi M, Gartel AL. Thiazole antibiotics target FoxM1 and induce apoptosis in human cancer cells. PLOS ONE. 2009;4(5):e5592.

23. Lam EW, Brosens JJ, Gomes AR, Koo CY. Forkhead box proteins: tuning forks for transcriptional harmony. Nat Rev Cancer. 2013;13(7):482-495.

24. Bellacosa A, et al. Akt activation by growth factors is a multiple-step process: the role of the $\mathrm{PH}$ domain. Oncogene. 1998;17(3):313-325.

25. Wilborn J, Crofford LJ, Burdick MD, Kunkel SL, Strieter RM, Peters-Golden M. Cultured lung fibroblasts isolated from patients with idiopathic pulmonary fibrosis have a diminished capacity to synthesize prostaglandin E2 and to express cyclooxygenase-2. J Clin Invest. 1995;95(4):1861-1868.

26. Moore BB, et al. Bleomycin-induced E prostanoid receptor changes alter fibroblast responses to prostaglandin E2. J Immunol. 2005;174(9):5644-5649.

27. White ES, et al. Prostaglandin E(2) inhibits fibroblast migration by E-prostanoid 2 receptormediated increase in PTEN activity. Am J Respir Cell Mol Biol. 2005;32(2):135-141.

28. Nho RS, Peterson M, Hergert P, Henke CA. FoxO3a (Forkhead Box O3a) deficiency protects idiopathic pulmonary fibrosis (IPF) fibroblasts from type I polymerized collagen matrix-induced apoptosis via caveolin-1 (cav-1) and Fas. PLoS ONE. 2013;8(4):e61017.

29. Gomes AR, Zhao F, Lam EW. Role and regulation of the forkhead transcription factors FOXO3a and FOXM1 in carcinogenesis and drug resistance. Chin J Cancer. 2013;32(7):365-370.

30. Schachter TN, Shen T, Liu Y, Schneider MF. Kinetics of nuclear-cytoplasmic translocation of Foxo1 and Foxo3A in adult skeletal muscle fibers. $A m J$ Physiol, Cell Physiol. 2012;303(9):C977-C990.
31. Bühling F, et al. Altered expression of membranebound and soluble CD95/Fas contributes to the resistance of fibrotic lung fibroblasts to FasL induced apoptosis. Respir Res. 2005;6:37.

32. Horowitz JC, et al. Survivin expression induced by endothelin-1 promotes myofibroblast resistance to apoptosis. Int J Biochem Cell Biol. 2012;44(1):158-169.

33. Moeller A, Ask K, Warburton D, Gauldie J, Kolb M. The bleomycin animal model: a useful tool to investigate treatment options for idiopathic pulmonary fibrosis? Int J Biochem Cell Biol. 2008;40(3):362-382.

34. Wang $\mathrm{M}$, et al. Combination treatment with bortezomib and thiostrepton is effective against tumor formation in mouse models of DEN/ PB-induced liver carcinogenesis. Cell Cycle. 2012;11(18):3370-3372.

35. Semren N, et al. Regulation of 26S Proteasome Activity in Pulmonary Fibrosis. Am J Respir Crit Care Med. 2015;192(9):1089-1101.

36. Bolte C, et al. Postnatal ablation of Foxm 1 from cardiomyocytes causes late onset cardiac hypertrophy and fibrosis without exacerbating pressure overload-induced cardiac remodeling. PLoS ONE. 2012;7(11):e48713.

37. Balli $D$, et al. Foxm 1 transcription factor is required for lung fibrosis and epithelial-to-mesenchymal transition. EMBO J. 2013;32(2):231-244.

38. Hung $C$, et al. Role of lung pericytes and resident fibroblasts in the pathogenesis of pulmonary fibrosis. Am J Respir Crit Care Med. 2013;188(7):820-830.

39. Rock JR, et al. Multiple stromal populations contribute to pulmonary fibrosis without evidence for epithelial to mesenchymal transition. Proc Natl Acad Sci USA. 2011;108(52):E1475-E1483.

40. Xie T, et al. Transcription factor TBX4 regulates myofibroblast accumulation and lung fibrosis. JClin Invest. 2016;126(8):3063-3079.

41. Vancheri C, Failla M, Crimi N, Raghu G. Idiopathic pulmonary fibrosis: a disease with similarities and links to cancer biology. Eur Respir J. 2010;35(3):496-504.

42. Selman M, Pardo A. Idiopathic pulmonary fibrosis: an epithelial/fibroblastic cross-talk disorder. Respir Res. 2002;3:3.

43. Hinz B. Formation and function of the myofibroblast during tissue repair. Jinvest Dermatol. 2007;127(3):526-537.

44. Penke LR, Huang SK, White ES, Peters-Golden M. Prostaglandin E2 inhibits $\alpha$-smooth muscle actin transcription during myofibroblast differentiation via distinct mechanisms of modulation of serum response factor and myocardinrelated transcription factor-A. J Biol Chem. 2014;289(24):17151-17162.

45. Sandbo N, Kregel S, Taurin S, Bhorade S, Dulin NO. Critical role of serum response factor 
in pulmonary myofibroblast differentiation induced by TGF-beta. Am J Respir Cell Mol Biol. 2009;41(3):332-338.

46. Gauthier-Rouvière C, Cavadore JC, Blanchard JM, Lamb NJ, Fernandez A. p67SRF is a constitutive nuclear protein implicated in the modulation of genes required throughout the G1 period. Cell Regul. 1991;2(7):575-588.

47. Gualdrini F, Esnault C, Horswell S, Stewart A, Matthews N, Treisman R. SRF co-factors control the balance between cell proliferation and contractility. Mol Cell. 2016;64(6):1048-1061.

48. Sisson TH, et al. Increased survivin expression contributes to apoptosis-resistance in IPF fibroblasts. Adv Biosci Biotechnol. 2012;3(6A):657-664.

49. Lama V, Moore BB, Christensen P, Toews GB, Peters-Golden M. Prostaglandin E2 synthesis and suppression of fibroblast proliferation by alveolar epithelial cells is cyclooxygenase2-dependent. Am J Respir Cell Mol Biol. 2002;27(6):752-758.

50. Huang SK, et al. Prostaglandin E(2) induces fibroblast apoptosis by modulating multiple survival pathways. FASEB J. 2009;23(12):4317-4326.

51. Kolodsick JE, Peters-Golden M, Larios J, Toews GB, Thannickal VJ, Moore BB. Prostaglandin E2 inhibits fibroblast to myofibroblast transition via E. prostanoid receptor 2 signaling and cyclic adenosine monophosphate elevation. Am J Respir Cell Mol Biol. 2003;29(5):537-544.

52. Stoyanova T, et al. p21 cooperates with DDB2 protein in suppression of ultraviolet ray-induced skin malignancies. J Biol Chem. 2012;287(5):3019-3028.

53. Ogawa R, Streiff MB, Bugayenko A, Kato GJ. Inhibition of PDE4 phosphodiesterase activity induces growth suppression, apoptosis, glucocorticoid sensitivity, p53, and p21(WAF1/CIP1) proteins in human acute lymphoblastic leukemia cells. Blood. 2002;99(9):3390-3397.

54 . Hodges RJ, et al. Severity of lung injury in cyclooxygenase-2-deficient mice is dependent on reduced prostaglandin $\mathrm{E}$ (2) production. Am J Pathol. 2004;165(5):1663-1676.

55. Moore BB, et al. GM-CSF regulates bleomycininduced pulmonary fibrosis via a prostaglandin-dependent mechanism. J Immunol. 2000;165(7):4032-4039.

56. Huang SK, et al. Variable prostaglandin E2 resistance in fibroblasts from patients with usual interstitial pneumonia. Am J Respir Crit Care Med. 2008;177(1):66-74.

57. Tsukui $\mathrm{T}$, et al. Qualitative rather than quantitative changes are hallmarks of fibroblasts in bleomycin-induced pulmonary fibrosis. Am J Pathol. 2013;183(3):758-773.

58. Fernandez IE, Eickelberg O. New cellular and molecular mechanisms of lung injury and fibrosis in idiopathic pulmonary fibrosis. Lancet. 2012;380(9842):680-688.

59. Gartel AL. Thiostrepton, proteasome inhibitors and FOXM1. Cell Cycle. 2011;10(24):4341-4342.

60. Radhakrishnan SK, Bhat UG, Hughes DE, Wang IC, Costa RH, Gartel AL. Identification of a chemical inhibitor of the oncogenic transcription factor forkhead box M1. Cancer Res. 2006;66(19):9731-9735.

61. Chen Y, Ruben EA, Rajadas J, Teng NN. In silico investigation of FOXM1 binding and novel inhibitors in epithelial ovarian cancer. Bioorg Med Chem. 2015;23(15):4576-4582.

62. Gormally MV, et al. Suppression of the FOXM1 transcriptional programme via novel small molecule inhibition. Nat Commun. 2014;5:5165.

63. Huang M, et al. IL-7 inhibits fibroblast TGF-beta production and signaling in pulmonary fibrosis. JClin Invest. 2002;109(7):931-937.

64. Kumar RK, O'Grady R, Maronese SE, Wilson MR. Epithelial cell-derived transforming growth factor-beta in bleomycin-induced pulmonary injury. Int J Exp Pathol. 1996;77(3):99-107.

65. Martinez FO, Gordon S. The M1 and M2 paradigm of macrophage activation: time for reassessment. F100OPrime Rep. 2014;6:13.

66. Wei Y, et al. Fibroblast-specific inhibition of TGF- $\beta 1$ signaling attenuates lung and tumor fibrosis. J Clin Invest. 2017;127(10):3675-3688.

67. Lech M, Anders HJ. Macrophages and fibrosis: How resident and infiltrating mononuclear phagocytes orchestrate all phases of tissue injury and repair. Biochim Biophys Acta. 2013;1832(7):989-997.
68. Vittal R, et al. Modulation of prosurvival signaling in fibroblasts by a protein kinase inhibitor protects against fibrotic tissue injury. Am J Pathol. 2005;166(2):367-375.

69. Hu B, Wu Z, Nakashima T, Phan SH. Mesenchymal-specific deletion of C/EBP $\beta$ suppresses pulmonary fibrosis. Am J Pathol. 2012;180(6):2257-2267.

70. Krupczak-Hollis K, et al. The mouse Forkhead Box $\mathrm{m} 1$ transcription factor is essential for hepatoblast mitosis and development of intrahepatic bile ducts and vessels during liver morphogenesis. Dev Biol. 2004;276(1):74-88.

71. Madisen L, et al. A robust and high-throughput Cre reporting and characterization system for the whole mouse brain. Nat Neurosci. 2010;13(1):133-140.

72. Huang SK, Scruggs AM, McEachin RC, White ES, Peters-Golden M. Lung fibroblasts from patients with idiopathic pulmonary fibrosis exhibit genome-wide differences in DNA methylation compared to fibroblasts from nonfibrotic lung. PLOS ONE. 2014;9(9):e107055.

73. Garrison G, et al. Reversal of myofibroblast differentiation by prostaglandin E(2). Am J Respir Cell Mol Biol. 2013;48(5):550-558.

74. Solinet S, Vitale ML. Isoform B of myosin II heavy chain mediates actomyosin contractility during TNFalpha-induced apoptosis. JCell Sci. 2008;121(Pt 10):1681-1692.

75. Swaney JS, et al. A novel, orally active LPA(1) receptor antagonist inhibits lung fibrosis in the mouse bleomycin model. Br JPharmacol. 2010;160(7):1699-1713.

76. Sisson TH, et al. Inhibition of myocardin-related transcription factor/serum response factor signaling decreases lung fibrosis and promotes mesenchymal cell apoptosis. Am J Pathol. 2015;185(4):969-986.

77. Ashcroft T, Simpson JM, Timbrell V. Simple method of estimating severity of pulmonary fibrosis on a numerical scale. JClin Pathol. 1988;41(4):467-470.

78. Hübner RH, et al. Standardized quantification of pulmonary fibrosis in histological samples. BioTechniques. 2008;44(4):507-511, 514. 